\title{
Solar neutrino results in Super-Kamiokande-III
}

K. Abe,${ }^{1}$ Y. Hayato, ${ }^{1,3}$ T. Iida, ${ }^{1}$ M. Ikeda,${ }^{1, \dagger}$ C. Ishihara, ${ }^{1}$ K. Iyogi,${ }^{1}$ J. Kameda,,${ }^{1}$ K. Kobayashi, ${ }^{1}$ Y. Koshio, ${ }^{1}$ Y. Kozuma, ${ }^{1}$ M. Miura, ${ }^{1}$ S. Moriyama,${ }^{1,3}$ M. Nakahata,,${ }^{1,3}$ S. Nakayama, ${ }^{1}$ Y. Obayashi, ${ }^{1}$ H. Ogawa, ${ }^{1}$ H. Sekiya,${ }^{1}$ M. Shiozawa, ${ }^{1,3}$ Y. Suzuki, ${ }^{1,3}$ A. Takeda, ${ }^{1}$ Y. Takenaga, ${ }^{1}$ K. Ueno, ${ }^{1}$ K. Ueshima, ${ }^{1}$ H. Watanabe,${ }^{1}$ S. Yamada, ${ }^{1}$ T. Yokozawa, ${ }^{1}$ S. Hazama, ${ }^{2}$ H. Kaji, ${ }^{2}$ T. Kajita, ${ }^{2,3}$ K. Kaneyuki, ${ }^{2,3}$ T. McLachlan, ${ }^{2}$ K. Okumura, ${ }^{2}$ Y. Shimizu, ${ }^{2}$

N. Tanimoto, ${ }^{2}$ M. R. Vagins, ${ }^{3,7}$ L. Labarga, ${ }^{4}$ L. M. Magro, ${ }^{4}$ F. Dufour, ${ }^{5}$ E. Kearns, ${ }^{5,3}$ M. Litos, ${ }^{5}$ J. L. Raaf, ${ }^{5}$ J. L. Stone,${ }^{5,3}$ L. R. Sulak, ${ }^{5}$ W. Wang, ${ }^{5,}$ M. Goldhaber, ${ }^{6}$ K. Bays, ${ }^{7}$ D. Casper, ${ }^{7}$ J. P. Cravens, ${ }^{7}$ W. R. Kropp, ${ }^{7}$ S. Mine, ${ }^{7}$ C. Regis, ${ }^{7}$ A. Renshaw, ${ }^{7}$ M. B. Smy, ${ }^{7,3}$ H. W. Sobel, ${ }^{7,3}$ K. S. Ganezer, ${ }^{8}$ J. Hill, ${ }^{8}$ W. E. Keig, ${ }^{8}$ J. S. Jang, ${ }^{9}$ J. Y. Kim, ${ }^{9}$ I. T. Lim, ${ }^{9}$ J. Albert, ${ }^{10}$ R. Wendell, ${ }^{10}$ T. Wongjirad, ${ }^{10}$ K. Scholberg, ${ }^{10,3}$ C. W. Walter, ${ }^{10,3}$ T. Ishizuka, ${ }^{11}$ S. Tasaka, ${ }^{12}$ J. G. Learned, ${ }^{13}$ S. Matsuno, ${ }^{13}$ Y. Watanabe, ${ }^{14}$ T. Hasegawa, ${ }^{15}$ T. Ishida, ${ }^{15}$ T. Ishii, ${ }^{15}$ T. Kobayashi, ${ }^{15}$ T. Nakadaira, ${ }^{15}$ K. Nakamura, ${ }^{15,3}$ K. Nishikawa,${ }^{15}$ H. Nishino, ${ }^{15}$ Y. Oyama,${ }^{15}$ K. Sakashita, ${ }^{15}$ T. Sekiguchi,${ }^{15}$ T. Tsukamoto,${ }^{15}$ A. T. Suzuki, ${ }^{16}$ Y. Takeuchi, ${ }^{16,3}$ A. Minamino, ${ }^{17}$ T. Nakaya, ${ }^{17,3}$ Y. Fukuda,${ }^{18}$ Y. Itow, ${ }^{19}$ G. Mitsuka, ${ }^{19}$ T. Tanaka, ${ }^{19}$ C. K. Jung, ${ }^{20}$ G. Lopez,${ }^{20}$ C. McGrew,${ }^{20}$ R. Terri, ${ }^{20}$ C. Yanagisawa, ${ }^{20}$ N. Tamura, ${ }^{21}$ H. Ishino, ${ }^{22}$ A. Kibayashi, ${ }^{22}$ S. Mino, ${ }^{22}$ T. Mori, ${ }^{22}$ M. Sakuda, ${ }^{22}$ H. Toyota ${ }^{22}$ Y. Kuno, ${ }^{23}$ M. Yoshida ${ }^{23}$ S. B. Kim, ${ }^{24}$ B. S. Yang, ${ }^{24}$ T. Ishizuka, ${ }^{25}$

H. Okazawa, ${ }^{26}$ Y. Choi,${ }^{27}$ K. Nishijima, ${ }^{28}$ Y. Yokosawa,${ }^{28}$ M. Koshiba, ${ }^{29}$ Y. Totsuka, ${ }^{29}, *$ M. Yokoyama,${ }^{29}$ S. Chen, ${ }^{30}$ Y. Heng, ${ }^{30}$ Z. Yang, ${ }^{30}$ H. Zhang, ${ }^{30}$ D. Kielczewska, ${ }^{31}$ P. Mijakowski, ${ }^{31}$ K. Connolly, ${ }^{32}$ M. Dziomba ${ }^{32}$ E. Thrane, ${ }^{32,8}$ and R. J. Wilkes ${ }^{32}$

(Super-Kamiokande Collaboration)

\footnotetext{
${ }^{1}$ Kamioka Observatory, Institute for Cosmic Ray Research, University of Tokyo, Kamioka, Gifu 506-1205, Japan

${ }^{2}$ Research Center for Cosmic Neutrinos, Institute for Cosmic Ray Research, University of Tokyo, Kashiwa, Chiba 277-8582, Japan

${ }^{3}$ Institute for the Physics and Mathematics of the Universe, University of Tokyo, Kashiwa, Chiba 277-8582, Japan

${ }^{4}$ Department of Theoretical Physics, University Autonoma Madrid, 28049 Madrid, Spain

${ }^{5}$ Department of Physics, Boston University, Boston, Massachusetts 02215, USA

${ }^{6}$ Physics Department, Brookhaven National Laboratory, Upton, New York 11973, USA

${ }^{7}$ Department of Physics and Astronomy, University of California, Irvine, Irvine, California 92697-4575, USA

${ }^{8}$ Department of Physics, California State University, Dominguez Hills, Carson, California 90747, USA

${ }^{9}$ Department of Physics, Chonnam National University, Kwangju 500-757, Korea

${ }^{10}$ Department of Physics, Duke University, Durham, North Carolina 27708, USA

${ }^{11}$ Junior College, Fukuoka Institute of Technology, Fukuoka, Fukuoka 811-0214, Japan

${ }^{12}$ Department of Physics, Gifu University, Gifu, Gifu 501-1193, Japan

${ }^{13}$ Department of Physics and Astronomy, University of Hawaii, Honolulu, Hawaii 96822, USA

${ }^{14}$ Physics Division, Department of Engineering, Kanagawa University, Kanagawa, Yokohama 221-8686, Japan

${ }^{15}$ High Energy Accelerator Research Organization (KEK), Tsukuba, Ibaraki 305-0801, Japan

${ }^{16}$ Department of Physics, Kobe University, Kobe, Hyogo 657-8501, Japan

${ }^{17}$ Department of Physics, Kyoto University, Kyoto, Kyoto 606-8502, Japan

${ }^{18}$ Department of Physics, Miyagi University of Education, Sendai, Miyagi 980-0845, Japan

${ }^{19}$ Solar Terrestrial Environment Laboratory, Nagoya University, Nagoya, Aichi 464-8602, Japan

${ }^{20}$ Department of Physics and Astronomy, State University of New York at Stony Brook, Stony Brook, New York 11794-3800, USA

${ }^{21}$ Department of Physics, Niigata University, Niigata, Niigata 950-2181, Japan

${ }^{22}$ Department of Physics, Okayama University, Okayama, Okayama 700-8530, Japan

${ }^{23}$ Department of Physics, Osaka University, Toyonaka, Osaka 560-0043, Japan

${ }^{24}$ Department of Physics, Seoul National University, Seoul 151-742, Korea

${ }^{25}$ Department of Systems Engineering, Shizuoka University, Hamamatsu, Shizuoka 432-8561, Japan

${ }^{26}$ Department of Informatics in Social Welfare, Shizuoka University of Welfare, Yaizu, Shizuoka, 425-8611, Japan

${ }^{27}$ Department of Physics, Sungkyunkwan University, Suwon 440-746, Korea

${ }^{28}$ Department of Physics, Tokai University, Hiratsuka, Kanagawa 259-1292, Japan

${ }^{29}$ The University of Tokyo, Bunkyo, Tokyo 113-0033, Japan
}

\footnotetext{
* Deceased.

${ }^{\dagger}$ Present address: Department of Physics, Kyoto University, Kyoto, Kyoto 606-8502, Japan.

${ }^{\ddagger}$ Present address: Department of Physics, University of Wisconsin-Madison, 1150 University Avenue, Madison, WI 53706, USA.

${ }^{\S}$ Present address: Department of Physics and Astronomy, University of Minnesota, Minneapolis, MN, 55455, USA.
} 
${ }^{30}$ Department of Engineering Physics, Tsinghua University, Beijing, 100084, China
${ }^{31}$ Institute of Experimental Physics, Warsaw University, 00-681 Warsaw, Poland
${ }^{32}$ Department of Physics, University of Washington, Seattle, Washington 98195-1560, USA

(Received 1 October 2010; published 24 March 2011)

\begin{abstract}
The results of the third phase of the Super-Kamiokande solar neutrino measurement are presented and compared to the first and second phase results. With improved detector calibrations, a full detector simulation, and improved analysis methods, the systematic uncertainty on the total neutrino flux is estimated to be $\pm 2.1 \%$, which is about two thirds of the systematic uncertainty for the first phase of Super-Kamiokande. The observed ${ }^{8} \mathrm{~B}$ solar flux in the 5.0 to $20 \mathrm{MeV}$ total electron energy region is $2.32 \pm 0.04$ (stat) \pm 0.05 (sys) $\times 10^{6} \mathrm{~cm}^{-2} \mathrm{sec}^{-1}$ under the assumption of pure electron-flavor content, in agreement with previous measurements. A combined oscillation analysis is carried out using SK-I, II, and III data, and the results are also combined with the results of other solar neutrino experiments. The best-fit oscillation parameters are obtained to be $\sin ^{2} \theta_{12}=0.30_{-0.01}^{+0.02}\left(\tan ^{2} \theta_{12}=\right.$ $\left.0.42_{-0.02}^{+0.04}\right)$ and $\Delta m_{21}^{2}=6.2_{-1.9}^{+1.1} \times 10^{-5} \mathrm{eV}^{2}$. Combined with KamLAND results, the best-fit oscillation parameters are found to be $\sin ^{2} \theta_{12}=0.31 \pm 0.01\left(\tan ^{2} \theta_{12}=0.44 \pm 0.03\right)$ and $\Delta m_{21}^{2}=$ $7.6 \pm 0.2 \times 10^{-5} \mathrm{eV}^{2}$. The ${ }^{8} \mathrm{~B}$ neutrino flux obtained from global solar neutrino experiments is $5.3 \pm$ 0.2 (stat + sys $) \times 10^{6} \mathrm{~cm}^{-2} \mathrm{~s}^{-1}$, while the ${ }^{8} \mathrm{~B}$ flux becomes $5.1 \pm 0.1(\mathrm{stat}+\mathrm{sys}) \times 10^{6} \mathrm{~cm}^{-2} \mathrm{~s}^{-1}$ by adding KamLAND results. In a three-flavor analysis combining all solar neutrino experiments, the upper limit of $\sin ^{2} \theta_{13}$ is 0.060 at $95 \%$ C.L.. After combination with KamLAND results, the upper limit of $\sin ^{2} \theta_{13}$ is found to be 0.059 at $95 \%$ C.L.
\end{abstract}

DOI: 10.1103/PhysRevD.83.052010

PACS numbers: $14.60 . P q$

\section{INTRODUCTION}

The third phase of Super-Kamiokande (SK-III) began in October 2006 and ended in August 2008 when the electronics were replaced. In SK-III, all 11129 photomultiplier tubes (PMT) have acrylic and fiber feinforced plastic (FRP) PMT covers (blast shields) which were added at the start of SK-II in order to protect against propagating shock waves from PMT implosions. In the inner detector, the active photodetector coverage is $40 \%$ (40\% in SK-I and $19 \%$ in SK-II). Thanks to detector improvements and superior analysis techniques, the SK-III's solar neutrino flux measurement is more precise than either SK-I's [1] (SK before the accident) or SK-II's [2] (SK with $46.5 \%$ of its PMTs) even with an exposure of only two years. In particular, the water purification system, event reconstruction and selection tools, as well as Monte Carlo detector simulation were improved. They will be explained in Sec. II and Sec. III in detail.

In Sec. IV, the results of oscillation analyses are presented. By adding SK-III data, it was found that the energy spectrum and the time variation of solar neutrinos obtained from our measurements favor only the large mixing angle solution (LMA) by constraining the ${ }^{8} \mathrm{~B}$ and hep neutrino flux to Sudbury Neutrino Observatory (SNO) neutral current flux $[3,4]$ and standard solar bodel (SSM) prediction [5], respectively. The first result of three-flavor neutrino oscillation analysis with the full SK data set will be shown as well as the two-flavor analysis result. In the last section of Sec. IV, the ${ }^{8} \mathrm{~B}$ flux value obtained from the results of all solar neutrino experiments (global solar analysis) will be shown to compare with the prediction of the SSM.

\section{SK-III PERFORMANCE}

\section{A. Water system}

A major background for the solar neutrino observation at SK is the radioactivity from radon $(\mathrm{Rn})$ from the $\mathrm{U} / \mathrm{Th}$ decay chain in the water. The water in the detector is made from natural mine water using a very high performance water purification system. Even though the water is extremely pure, there is still some $\mathrm{Rn}$ remaining. The $\mathrm{Rn}$ background events are very similar to solar events, so it is very difficult to remove them using only analysis tools. To reduce it, we have upgraded the system since the end of SK-I, including the addition of a new heat exchanger and two reverse osmosis units during the SK-II and III periods.

In addition, we investigated the water flow in the detector by intentionally injecting radon-enriched water. Tracing the resulting background events in time from this injected $\mathrm{Rn}$, we found stagnation of water in the top and bottom of the detector volume, which increased the background. To counter this effect, we installed new pipes and changed the water flow. Previously, the water was supplied from the bottom of the inner detector (ID) and drained from the top of both the ID and outer detector (OD). Now, it is supplied from the ID bottom and drained from the top and bottom in OD and the top in ID with a total flow of 60 tons/hr, which is 2 times faster than before. This final setting has been in effect since August, 2007. As a result of these improvements, we have a central region with half of SK-I's background, enabling a lowering of the energy threshold.

Note that the excessive background near the wall and bottom consisting of $\gamma$ rays due to the FRP cover also 
existed in SK-II. This background could not be reduced by improving the water system.

\section{B. Event Reconstruction}

\section{Vertex}

The event vertex reconstruction for solar neutrino analysis performs a maximum likelihood fit to the timing residuals of the Cherenkov signal as well as the dark noise background for each testing vertex [6]. The vertex reconstruction method in SK-III was initially installed in SK-II and further improved over SK-II. It now has better resolution than SK-I.

Figure 1 shows the vertex resolution for SK-III. The vertex resolution is defined as the distance from the true vertex position containing $68.3 \%$ of all reconstructed vertices. The vertex resolution in SK-I for $5 \mathrm{MeV}$ electrons is $125 \mathrm{~cm}$; here it is improved to $100 \mathrm{~cm}$.

A bias in vertex reconstruction is called the "vertex shift." The vertex shift is defined as the vector from the averaged vertex of reconstructed events to that of the corresponding simulated Monte Carlo (MC) events. Because the vertex shift results in events moving in or out of the fiducial volume, it represents one of the main systematic uncertainties for the solar neutrino flux measurement.

The vertex shift is measured by placing a Ni-Cf gammaray source [7] at several positions inside the detector (hereafter, the calibration using this Ni-Cf source is called "Ni calibration" or "Ni events"). The reconstructed data vertices at the fiducial volume edge were shifted more than $10 \mathrm{~cm}$ from the real source position inward toward the detector center, while those of the MC simulation were shifted less than $3 \mathrm{~cm}$. It was found that this shift in data was due to an electronic effect of the relative hit timing within a wide range $(\sim 100 \mathrm{nsec})$. We measured the timing linearity by artificially shifting the common stop signal of individual time-to-digital converters for each hit channel. We found that a correction of $-0.7 \%$ to the hit timing was required to restore linearity. After the correction was

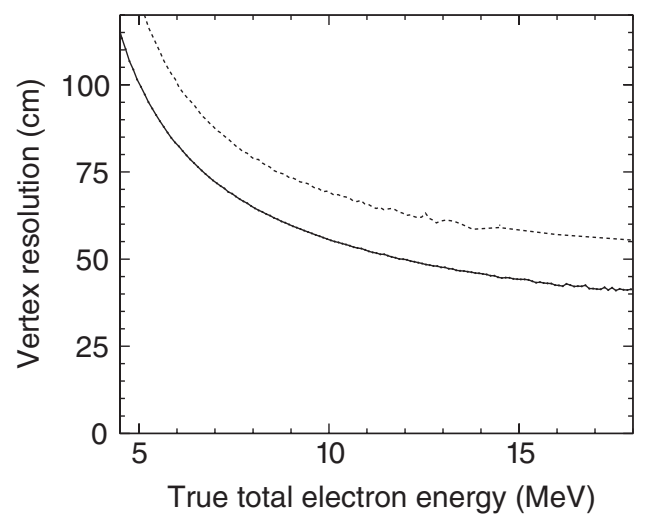

FIG. 1. Solid line shows the vertex resolution for SK-III as a function of the true total electron energy, while the dashed line shows that of SK-I.

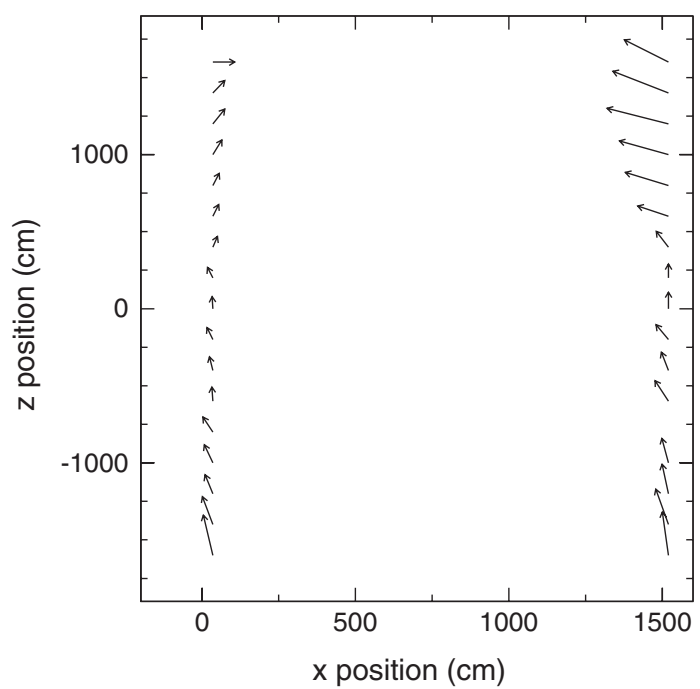

FIG. 2. Vertex shift of Ni calibration events. The origin of the arrows shows the true $\mathrm{Ni}$ source position and the direction indicates the averaged reconstructed position direction. The length of the arrow indicates the magnitude of the vertex shift. All vertex shifts are scaled by a factor of 20 to make them easier to see.

applied, the vertex shift shortened significantly. Figure 2 shows the vertex shift in SK-III with timing correction. The definition of $x$ and $z$ in Fig. 2 (and other variables) is explained in Fig. 3.

\section{Direction}

The direction reconstruction is based on the SK-I method: a likelihood function is used to compare Cherenkov ring patterns between data and MC simulation. An energy dependence is now included in the likelihood function for SK-III. The ring pattern distributions and their energy dependences are simulated for several energy ranges using electron $\mathrm{MC}$ simulation events.

Figure 4 shows the likelihood as a function of the reconstructed event energy and the opening angle between

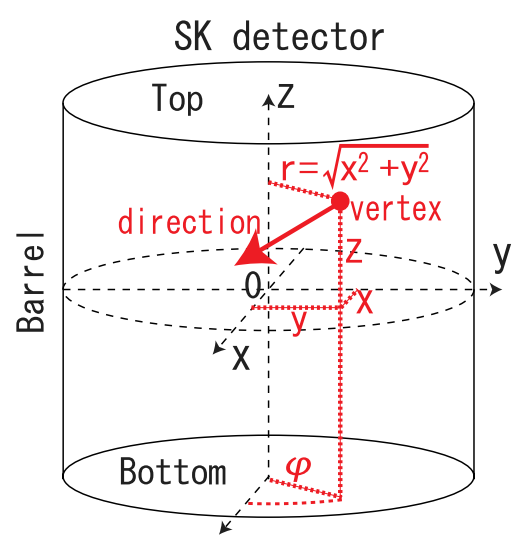

FIG. 3 (color online). Definition of detector coordinate system. 


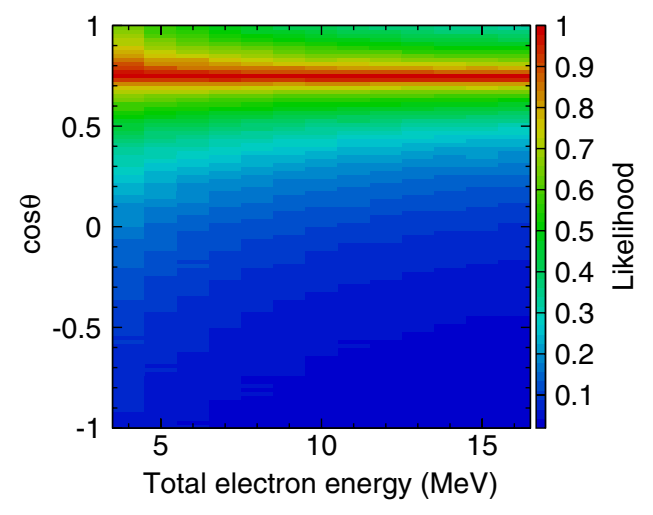

FIG. 4 (color online). Likelihood value for reconstruction of event direction as a function of the reconstructed total electron energy and opening angle between the reconstructed direction and the direction from vertex to each hit PMT.

the reconstructed direction and the direction from the vertex to each hit PMT. Figure 5 shows the absolute angular resolution, which is defined as the angle of the cone around the true direction containing $68.3 \%$ of the reconstructed directions. For SK-III, the angular resolution is improved compared to SK-I by about $10 \%$ at $10 \mathrm{MeV}$ and is close to the limit due to multiple Coulomb scattering of electrons. Note that the improvement of vertex reconstruction also contributes to the improvement of angular resolution, especially in the low energy region below $6.5 \mathrm{MeV}$.

\section{Energy}

The reconstruction of event energy is similar to that for SK-I [1]. The most important modifications with respect to SK-I are due to photocathode coverage and blast shields. Starting with the number of in-time hit PMTs $\left(N_{50}\right.$ coincident within $50 \mathrm{~ns}$ after the subtraction of time-of-flight (TOF) of Cherenkov photon from the reconstructed vertex to the hit PMT position), several corrections, described

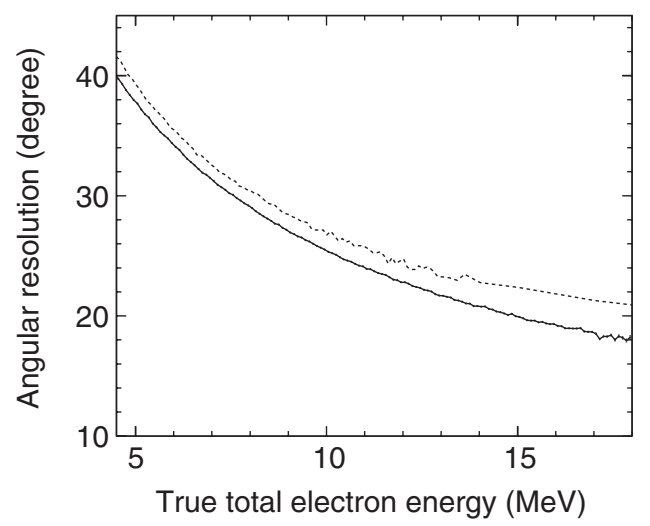

FIG. 5. The solid line shows the angular resolution of SK-III as a function of the true total electron energy, while the dashed line shows that of SK-I. below, are made. The resulting effective hit sum $N_{\text {eff }}$ has less position dependence than $N_{50}$. From $N_{\text {eff }}$, we determine energy. This procedure is further outlined in [1], and is also explained in [2].

The definition of $N_{\text {eff }}$ is:

$$
\begin{aligned}
N_{\text {eff }}= & \sum_{i}^{N_{50}}\left\{\left(X_{i}+\epsilon_{\text {tail }}-\epsilon_{\text {dark }}\right) \times \frac{N_{\text {all }}}{N_{\text {alive }}} \frac{1}{S\left(\theta_{i}, \phi_{i}\right)}\right. \\
& \left.\times \exp \left(\frac{r_{i}}{\lambda(t)}\right) \times G_{i}(t)\right\}
\end{aligned}
$$

where the explanations for the factors are as follows:

$X_{i}$ : This factor estimates the effect of multiple photoelectrons in the $i$-th hit PMT. If an event occurs close to a detector wall and is directed towards the same wall, the Cherenkov cone does not have much distance to expand, and the observed number of hits is small. The correction $X_{i}$ for this effect is defined as

$$
X_{i}= \begin{cases}\frac{\log \frac{1}{1-x_{i}}}{x_{i}}, & x_{i}<1 \\ 3.0, & x_{i}=1\end{cases}
$$

where $x_{i}$ is the ratio of hit PMTs in a $3 \times 3$ PMT region surrounding the $i$-th PMT to the total number of live PMTs in the same area. The $-\log \left(1-x_{i}\right)$ term is the estimated number of photons per one PMT in that area and is determined from Poisson statistics. When $x_{i}=1,3.0$ is assigned to $X_{i}$.

$\epsilon_{\text {tail }}:$ Some Cherenkov photons being scattered or reflected arrive late at the PMT, and make late hits outside the $50 \mathrm{nsec}$ time window. To correct the effect of the late hits, the term

$$
\epsilon_{\text {tail }}=\frac{N_{100}-N_{50}-N_{\text {alive }} \times R_{\text {dark }} \times 50 \mathrm{nsec}}{N_{50}}
$$

is added where $N_{100}$ is the maximum number of hits found by a 100 nsec sliding time window search.

$\epsilon_{\text {dark }}$ : This factor corrects for hits due to dark noise in the PMTs.

$$
\epsilon_{\mathrm{dark}}=\frac{50 \mathrm{nsec} \times N_{\mathrm{alive}} \times R_{\mathrm{dark}}}{N_{50}}
$$

where $N_{\text {alive }}$ is the number of all live inner detector (ID) PMTs and $R_{\text {dark }}$ is the measured dark rate for a given data-taking period.

$\frac{N_{\text {all }}}{N_{\text {alive }}}$ : This factor is for the time variation of the number of dead PMTs. $N_{\text {all }}$ is total number of PMTs; for SK-III it is 11129.

$\frac{1}{S\left(\theta_{i}, \phi_{i}\right)}$ : This factor accounts for the direction-dependent photocathode coverage. $S\left(\theta_{i}, \phi_{i}\right)$ is the effective photocathode area of the $i$-th hit PMT as viewed from the angles $\left(\theta_{i}, \phi_{i}\right)$ to take into account the shadowing of PMTs for glancing angles $\theta_{i}$. S is determined by MC simulation with the FRP PMT covers; the resulting $N_{\text {eff }}$ is checked by an 


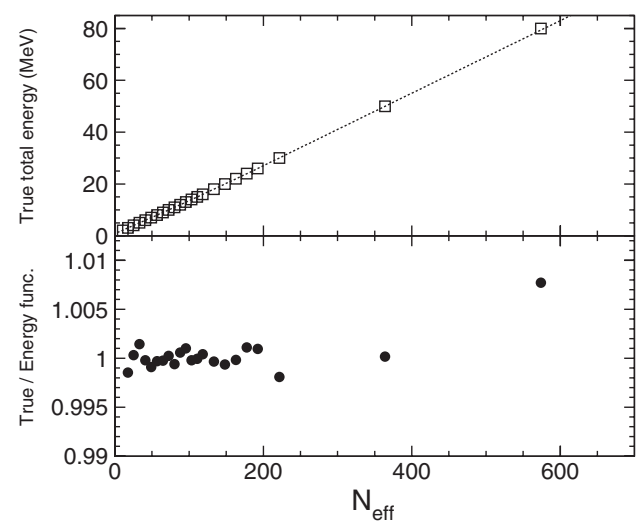

FIG. 6. Relation between $N_{\text {eff }}$ and the true total electron energy $(\mathrm{MeV})$ obtained from $\mathrm{MC}$ simulation. The dotted line in the upper figure shows the fitted polynomial function. The lower figure shows the deviation of the reconstructed energy from the polynomial function.

electron linear accelerator (LINAC) and an in-situ deuterium-tritium neutron generator (DT) calibration data. $\exp \left(\frac{r_{i}}{\lambda(t)}\right)$ : The water transparency is accounted for by this factor, where $r_{i}$ is the distance from the reconstructed vertex to the $i$-th hit PMT. $\lambda(t)$ is the measured water transparency for a given data-taking period.

$G_{i}(t)$ : This factor adjusts the relative quantum efficiency of the PMTs. The differences in the quantum efficiency depend on the fabrication date of the PMTs.

After determining $N_{\text {eff }}$, an event's energy in $\mathrm{MeV}$ can be calculated as a function of $N_{\text {eff }}$. The relation between $N_{\text {eff }}$ and $\mathrm{MeV}$ is obtained using monoenergetic electron $\mathrm{MC}$ simulated events as shown in Fig. 6. The conversion function from $N_{\text {eff }}$ to $\mathrm{MeV}$ is determined by fitting the relation with a fourth-order polynomial function for the lower energy region $(<25 \mathrm{MeV})$ and a first-order polynomial function for the higher energy region $(\geq 25 \mathrm{MeV})$.

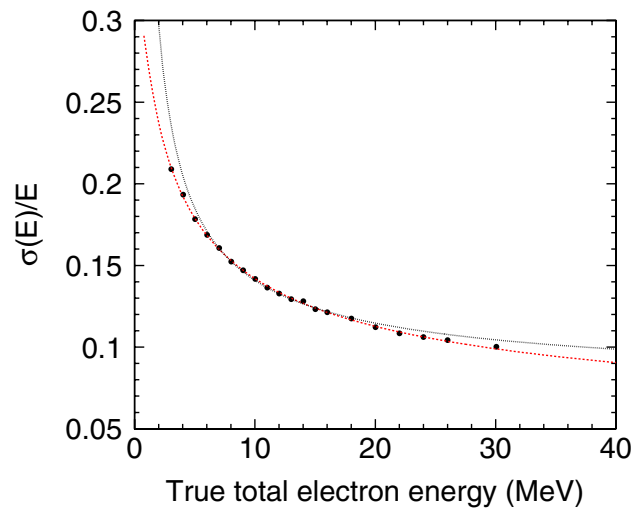

FIG. 7 (color online). Energy resolution function obtained by electron MC simulation. Black points show 1 standard deviation for a Gaussian fit of the MC simulation divided by the true total electron energy, while the red (dashed) line shows a fit to a polynomial function. The black (dotted) line shows SK-I energy resolution.
The systematic uncertainty of the reconstructed energy is checked by LINAC and DT calibration.

When calculating the energy for data events, the water transparency value, as determined by decay electrons from cosmic-ray muons, measured for six-day intervals, is used as an input parameter. For MC events, the change in water transparency and the relative quantum efficiency is simulated.

The detector's energy resolution is well described by a Gaussian function. The energy resolution is described by

$$
\sigma(E)=-0.123+0.376 \sqrt{E}+0.0349 E,
$$

in units of MeV (see Fig. 7). The SK-I resolution is $\sigma=$ $0.2468+0.1492 \sqrt{E}+0.0690 E$ which is shown also in Fig. 7. For low energy SK-III events, the energy resolution is improved by $5 \%$, which is mainly due to the improved vertex reconstruction.

\section{Energy calibration}

As for SK-I, the primary instrument for energy calibration in SK-III is LINAC. A detailed discussion of the LINAC calibration methods can be found elsewhere [8]. Single electrons are injected into the SK detector at various positions and at energies between 4.4 and $18.9 \mathrm{MeV}$. However, we could only take data with $4.4 \mathrm{MeV}$ electrons at two positions, because the tuning of the electron beam is difficult for the lower energies. For this reason we did not include the $4.4 \mathrm{MeV}$ data, and the lowest energy we included in this analysis is $4.7 \mathrm{MeV}$. The reconstructed energies of LINAC events are compared against those of the MC simulation to determine the energy scale. The absolute correction factor for PMT quantum efficiency was tuned to minimize any deviation between data and MC. The effect of the water transparency change on the energy scale was estimated as $0.22 \%$ by averaging over all energies and positions. The uncertainty of the electron beam energy, determined by a Ge detector measurement, is $0.21 \%$ (the same as for SK-I).

In addition to the LINAC calibration, energy scale calibration is done using ${ }^{16} \mathrm{~N}$ produced with DT [9]. The generated 14.2 MeV neutrons exchange their charge with ${ }^{16} \mathrm{O}$. The produced ${ }^{16} \mathrm{~N}$ decays into $\beta$ and $\gamma$ with a half-life of 7.13 seconds. With a $Q$-value of $10.4 \mathrm{MeV},{ }^{16} \mathrm{~N}$ most probably decays into ${ }^{16} \mathrm{O}$, an electron with maximum energy $4.3 \mathrm{MeV}$ and a $\gamma$ ray of energy $6.1 \mathrm{MeV}$. The peak value of the energy distribution is taken to evaluate the energy scale. DT data-taking is faster than LINAC datataking, so more positions can be checked.

The position dependence of the energy scale systematic uncertainty was estimated using only LINAC calibration data for SK-I and II, while for SK-III, in addition DT calibration data are used to take into account the $z$ dependence and $\phi$ dependence of the energy scale. The $z$ dependence is measured by LINAC, and the $\phi$ dependence is measured by DT. The LINAC can only take data at 


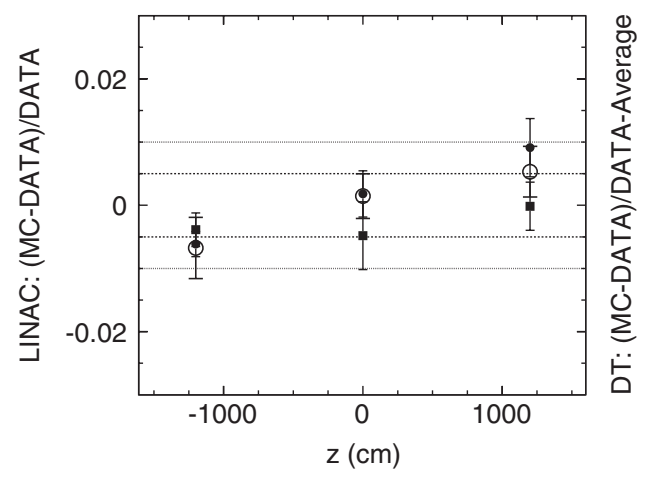

FIG. 8. The $z$ dependence of the energy scale measured by LINAC calibration. The marker shows an average of (MCDATA)/DATA over four energies at each data-taking point. The error bars show the root mean square of (MC-DATA)/ DATA for four energies. The filled circle markers are for $R=$ $4 \mathrm{~m}(x=-4 \mathrm{~m}, y=0 \mathrm{~m})$ and the square markers are for $r=$ $12 \mathrm{~m}(x=-12 \mathrm{~m}, y=0 \mathrm{~m})$. The open circle markers show the $z$ dependence obtained by DT calibration. The dashed and dotted lines show \pm 1 and $0.5 \%$, respectively. The edge of the fiducial volume is the same as the edge of the plot window (from $-1610 \mathrm{~cm}$ to $1610 \mathrm{~cm}$ ).

$\phi=180$ degrees, whereas the DT generator can take data at five different positions at the same $z$ position: center ( $r=4 \mathrm{~m}), \phi=0,90,180$, and 270 degrees $(r=12 \mathrm{~m})$.

Figure 8 shows the difference of the energy scale between LINAC data and LINAC MC as a function of LINAC position. By averaging over all positions, the $z$ dependence is estimated to be $0.06 \%$ (the difference between $r=4 \mathrm{~m}$ and $r=12 \mathrm{~m}$ is also included here.)

Figure 9 shows the difference of energy scale between DT data and DT MC. The vertical axis is normalized by the average of measurements at the same $z$ positions. The

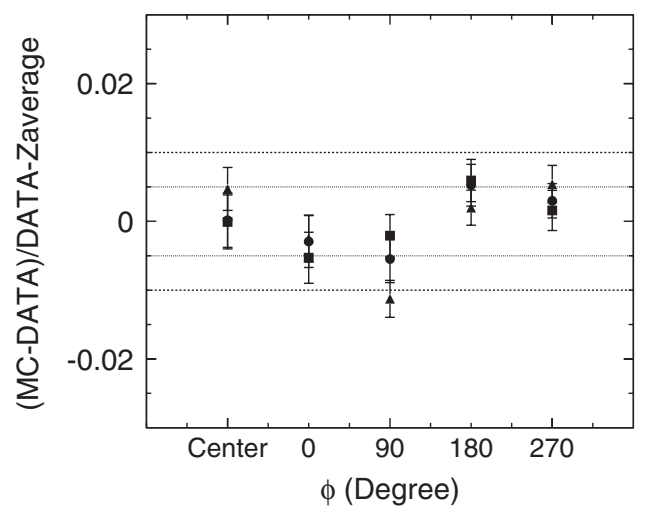

FIG. 9. The $\phi$ dependence of the energy scale measured by DT calibration. The marker shows (MC-DATA)/DATA for each point and the error bars show statistical uncertainty. The circle, square, and triangle markers are for $z=+12 \mathrm{~m}, 0 \mathrm{~m}$, and $-12 \mathrm{~m}$, respectively. Zaverage is the average difference of energy scale between MC simulation and DT data obtained at the same $z$-position. The dashed and dotted lines show \pm 1 and $0.5 \%$, respectively.

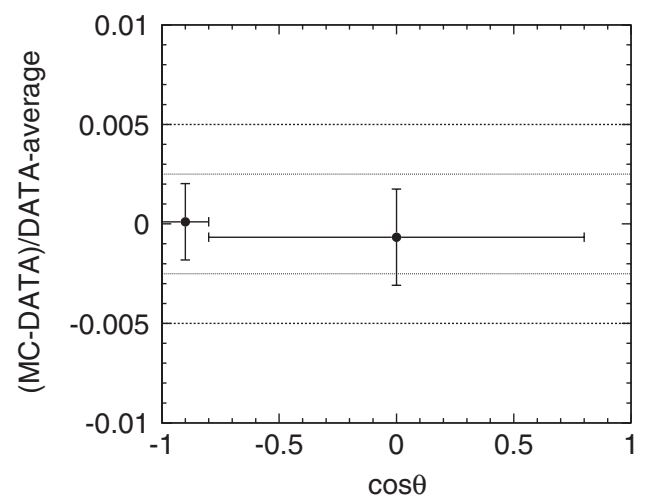

FIG. 10. Energy scale difference between LINAC direction and other direction measured by DT calibration (LINAC direction range includes $76 \%$ of $7 \mathrm{MeV}$ electrons). The solid and dotted lines show \pm 1 and $0.5 \%$, respectively.

mean value of the deviation from the average is taken as the $\phi$ dependence of energy scale, which is $0.35 \%$. A resulting uncertainty of $\pm 0.35 \%$ for the overall position dependence is estimated.

${ }^{16} \mathrm{~N}$ decays allow directional studies of the energy scale which are not possible with the LINAC beam. The observed energy at several positions in the detector is compared with the MC-simulated energy and the difference is shown to agree with values obtained from LINAC data and MC. The ${ }^{16} \mathrm{~N}$ energy scale difference between LINAC direction (downward direction) and the average of the other directions is estimated as $\pm 0.25 \%$, as shown in Fig. 10. This difference is taken as the directional uncertainty of the energy scale.

Finally, the energy scale uncertainty is calculated to be $0.53 \%$ which is summarized in Table I. This is slightly smaller than the SK-I estimated value of $0.64 \%$.

Energy resolutions of LINAC events are also compared for data and MC simulation. Figure 11 shows the difference of the energy resolution between data and $\mathrm{MC}$ as a function of the total electron energy. From Fig. 11, $\pm 2.5 \%$ systematic uncertainty is assigned to energy-correlated systematic uncertainty for the spectrum measurement.

Quantitative estimates of trigger efficiencies are also obtained from ${ }^{16} \mathrm{~N}$ data. The lowest hardware threshold setting has been in effect since April 2008. At this setting, the SK-III trigger achieved more than $99 \%$ efficiency at 4.5 MeV total electron energy. Before this time, the trigger efficiency was more than $99 \%$ at $5.0 \mathrm{MeV}$ total electron energy. Figure 12 shows the trigger efficiencies of the lowest threshold period in SK-III.

TABLE I. Systematic uncertainty of the energy scale.

\begin{tabular}{ll}
\hline \hline Position dep. & $0.35 \%$ \\
Direction dep. & $0.25 \%$ \\
Water transparency & $0.22 \%$ \\
LINAC energy sys. & $0.21 \%$ \\
Total & $0.53 \%$ \\
\hline \hline
\end{tabular}




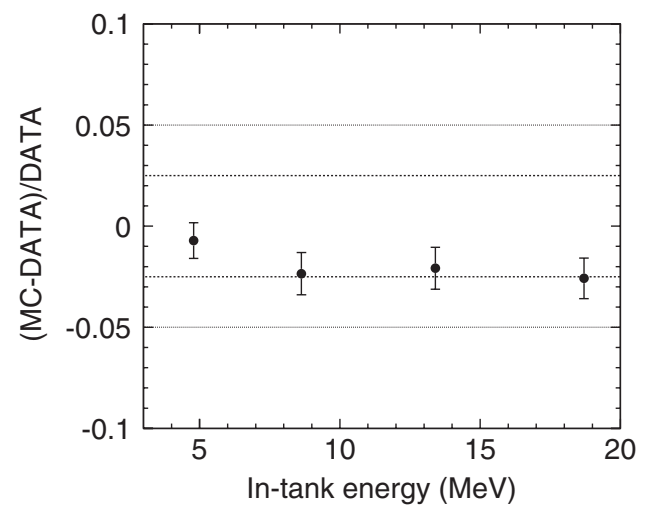

FIG. 11. Energy resolution difference between MC simulation and data as a function of energy, obtained by LINAC calibration.

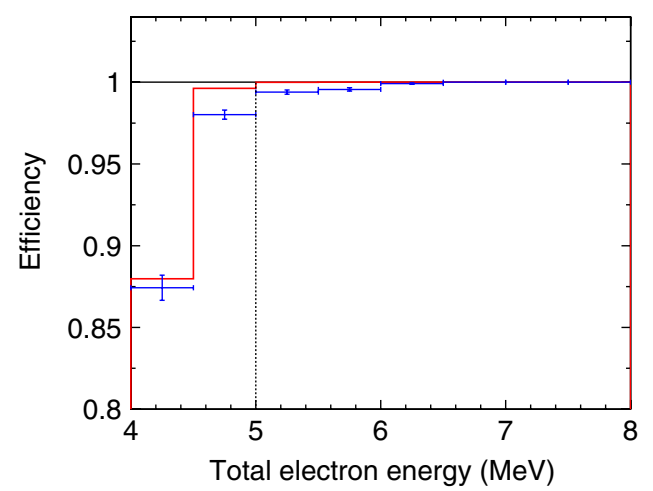

FIG. 12 (color online). Trigger efficiency as a function of energy. Markers are ${ }^{16} \mathrm{~N}$ calibration data and the solid histogram is MC simulation. The vertical dashed line shows the analysis threshold, $5.0 \mathrm{MeV}$.

\section{Optical calibration \\ 1. Light propagation in water}

For light propagation in water, a three-part model of light propagation consisting of absorption and two kinds of scattering is adopted for SK-III (as well as both SK-I and SK-II). In contrast to the previous phases, for SK-III the models are tuned using nitrogen/dye laser calibration. We measured the attenuation length of scattering and absorption for four wavelengths $(337,365,400$ and $420 \mathrm{~nm})$ and tuned the water coefficients based on the measurement. The water coefficients are described by:

$$
\begin{gathered}
\alpha_{\text {ray }}(\lambda)=\frac{r_{1}}{\lambda^{4}}\left(r_{2}+\frac{r_{3}}{\lambda^{r_{4}}}\right) \quad \alpha_{\text {mie }}(\lambda)=\frac{m}{\lambda^{4}} \\
\alpha_{a b s}(\lambda)=\frac{a}{\lambda^{4}}+\alpha_{\text {long }}(\lambda, a)
\end{gathered}
$$

Based on the data of February 2007 and 2008, we determined $r_{i}, m$, and $a . \alpha_{\text {long }}(\lambda, a)$ was determined by a thirdparty independent measurement introduced in SK-II $[2,10]$. Near the $400 \mathrm{~nm}$ region, we modified the relation using nitrogen/dye laser calibration. The crossing point of the SK and independent measurements [11] is varied by

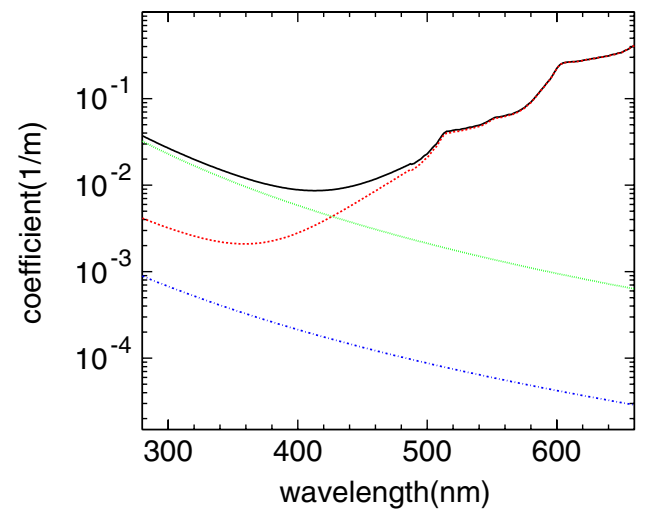

FIG. 13 (color online). Wavelength dependence of the water coefficients: scattering and absorption combined (black, solid line), absorption (red, dashed line), Rayleigh scattering (green, dotted line) and Mie scattering (blue, dashed-dotted line). The absorption coefficient is a function of water transparency and the water transparency in this graph is $139 \mathrm{~m}$.

another parameter $(a)$. The results of the determination of these parameters are shown in Fig. 13.

The time dependence and position dependence of the water quality are described in the following.

(a) Time Dependence. The time variations of the water coefficients and the water transparency are measured simultaneously by nitrogen/dye laser calibration and using decay electrons from cosmic-ray muons. These measurements confirmed that the change of the water transparency is mainly caused by the change of the absorption coefficient. We obtained the relation of the absorption coefficient and the water transparency in SK-III using those data as well as water transparency as measured by decay electrons from cosmic-ray muons, as for SK-I.

Figure 14 shows the time variation of the measured water transparency during SK-III and the stability of the peak energy of the decay electrons in SK-III as a function of time. The stability of the energy scale has $0.47 \%$ root mean square.

(b) Position Dependence. It was found that the PMT hit rates measured for a Ni-Cf gamma-ray source at the top region in the detector are systematically lower than those for the bottom region by $3 \sim 5 \%$. This rate difference is denoted the "top-bottom asymmetry" (TBA). The MC simulation cannot reproduce the top-bottom asymmetry with a uniform attenuation length throughout the detector volume. In order to solve this problem, a simple model of the light absorption is introduced to take into account the dependence of the attenuation length on depth. In this modeling, depth dependence of the absorption parameter is considered, because the dominant contribution to the time variation of the water transparency is the absorption. The depth dependence can be modeled as 


$$
\alpha_{a b s}(\lambda, z)= \begin{cases}\alpha_{a b s}(\lambda)(1+\beta \cdot z), & \text { for } z \geq-1200 \mathrm{~cm} \\ \alpha_{a b s}(\lambda)(1-\beta \cdot 1200), & \text { for } z<-1200 \mathrm{~cm}\end{cases}
$$

Below $z=-1200 \mathrm{~cm}$, the absorption coefficient is assumed to be uniform due to convection of the water. $\beta$ is called the TBA parameter (unit is $\mathrm{cm}^{-1}$ ) and it varies in time, because TBA varies. The $\beta$ for each period was tuned to minimize the TBA for that period.

Figure 15 shows a comparison of the hit-rate between data and $\mathrm{MC}$ simulation with the tuned $\beta$ value, which is $8.85 \times 10^{-5} \mathrm{~cm}^{-1}$, and without $\beta$ correction, respectively.

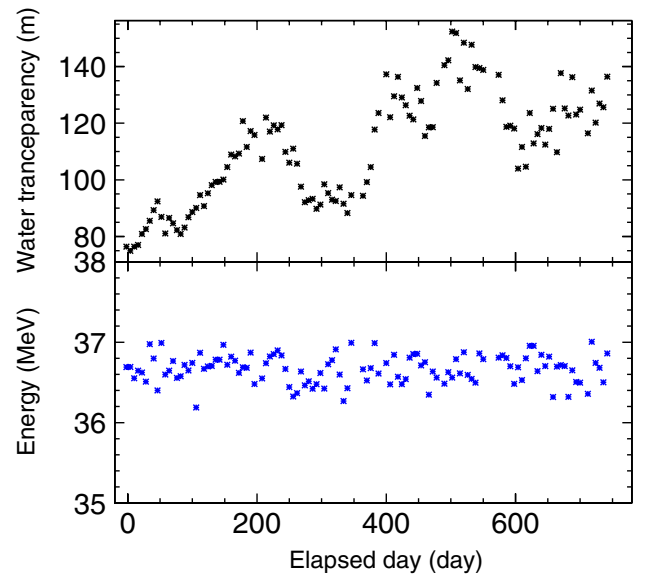

FIG. 14 (color online). The upper figure shows the time variation of the measured water transparency (weighted by the Cherenkov spectrum) during SK-III. The lower figure shows the stability of the peak energy of decay electrons in SK-III as a function of time.
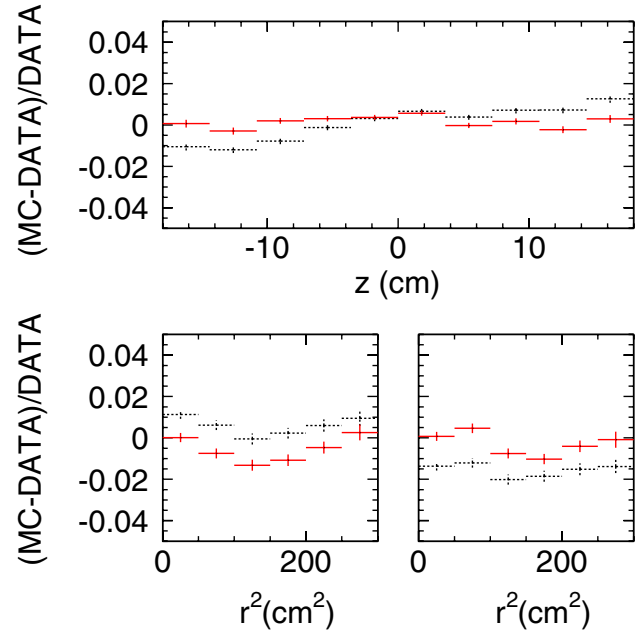

FIG. 15 (color online). Hit-rate difference, (MC-DATA)/ DATA, for barrel (upper), top (left lower), and bottom (right lower). Black (dashed line) shows $\beta=0$. Red (solid line) shows the hit-rate difference with tuned $\beta$. This calibration data was taken in February 2008, the same period as the LINAC calibration.
The hit rate is defined as the number of hits in each PMT in units of the averaged number of hits for all PMTs during the calibration run. As shown by the figure, data and MC show better agreement with the $\beta$ correction.

\section{Reflectivity of black sheet}

In the detector simulation, the reflectivity of the black sheet which covers the ID wall is calculated using the law of Fresnel, taking into account the polarization of Cherenkov photons. To better model the real situation, we measure the reflectivity for three incident angles using calibration data taken from a movable light injector and a black sheet reflector. We found that a value of half of the SK-I reflectivity gives better agreement and a wavelengthdependent correction is newly applied for SK-III.

In addition, to better describe the diffuse and specular reflection on the black sheet, we use two models for reflection: the Lambert model is used to describe diffuse reflection, and the Phong model is used for description of specular highlights [2].

\section{PMT and electronics}

The PMT must be simulated as precisely as possible. Reflection and quantum efficiency are tuned using the nitrogen/dye laser calibration data. In contrast to the description used for SK-I, we put a wavelength dependence into the reflection and an incident angle dependence into the quantum efficiency. The position and width of the PMT after-pulses and the timing resolution were tuned using data from single electrons injected into the SK detector by LINAC. After these tunings, the timing distributions of LINAC data and MC simulation agree very well, as shown in Fig. 16.

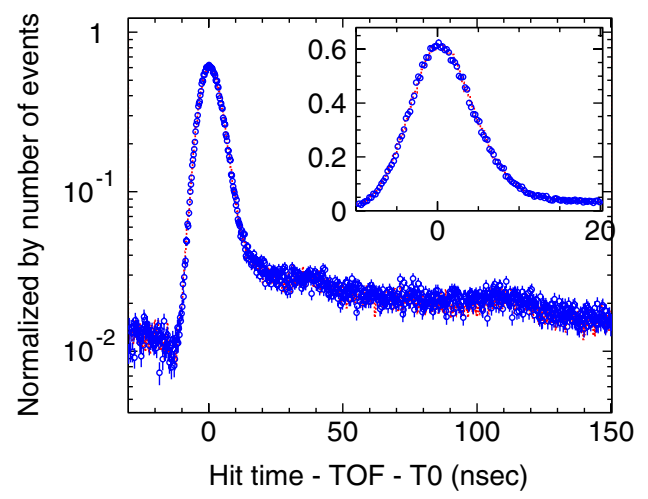

FIG. 16 (color online). Timing distribution of each hit PMT after tuning the MC simulation, for LINAC 5.1 MeV $(x, z)=$ $(-4 \mathrm{~m}, 0 \mathrm{~m})$ data. The blue histogram with open circles shows data and the red dashed histogram shows MC simulation. 


\section{DATA ANALYSIS}

\section{A. Data set}

As for SK-I and II, SK-III has two trigger levels for solar neutrino analysis: low-energy (LE) and super-low-energy (SLE) thresholds. SLE-triggered events are filtered online to reduce the amount of data written to limited storage space. Events reconstructed outside the fiducial volume are rejected. The data are reduced by a factor of approximately six. From Aug. 4, 2006 to Jan. 24, 2007, only the LE trigger threshold was applied. The LE trigger is $100 \%$ efficient above $6.5 \mathrm{MeV}$ and the live time of this period is 121.7 days. The threshold was lowered and the first SLE data were taken with more than $99 \%$ efficiency at $5.0 \mathrm{MeV}$ from Jan. 24, 2007 to Apr. 17, 2008. This period is called the SLE1 period, and the live time of this period is 331.5 days. From Apr. 17, 2008 to Aug. 18, 2008, a lower SLE trigger threshold was applied and the SLE trigger had more than $98 \%$ efficiency at $4.5 \mathrm{MeV}$. This period is called SLE2 period, and the live time of this period is 94.8 days.

Two neutrino samples are used for SK-III analysis. The first sample, for event energies between 6.5 and $20 \mathrm{MeV}$, has a total live time of 547.9 days. The second sample, for event energies between 5.0 and $6.5 \mathrm{MeV}$, has a total live time of 298.2 days after rejecting high background periods caused by radioactive impurities accidentally injected into the detector.

\section{B. Event selection}

Basic explanations of each selection step are as follows:

(i) Run selection

In this selection, short runs $(<5 \mathrm{~min}$.), runs with hardware and/or software problems, or calibration runs are rejected.

(ii) Cosmic-ray muon

Cosmic-ray muon events are rejected by a total charge cut $(<2000$ photo-electron (p.e.) in ID).

(iii) Electronic noise reduction

Events due to electronic noise are rejected.

(iv) Fiducial volume cut

Events which have reconstructed vertex position within $2 \mathrm{~m}$ of the ID wall are also rejected.

(v) Spallation cut

This cut is to reject events caused by cosmic-ray muons.

(vi) Event quality cut

In this step, results of event reconstructions are tested. In particular, (a) quality of vertex and direction reconstruction, and (b) hit pattern, (c) result of the second vertex reconstruction used for SK-I [1] are checked. Events produced by flasher PMTs are also rejected in this step.

(vii) External event cut

This cut is to reject events induced by radioactivity from the PMTs or the detector wall structure. (viii) Cosmogenic ${ }^{16} \mathrm{~N}$ cut

Events caused by decay of ${ }^{16} \mathrm{~N}$ are rejected. The

${ }^{16} \mathrm{~N}$ is produced when cosmic-ray $\mu$ is captured by ${ }^{16} \mathrm{O}$ in water.

(ix) Small clustered hit cut and tighter fiducial volume cut

These cuts reject events which have clustered hits (see III C and III D).

For more detailed descriptions of the reduction steps, please refer to $[1,2]$.

\section{Small clustered hit cut for the lowest energy region}

This cut is newly developed for SK-III to reduce the low energy background in regions near the edge in the detector. As described in the previous section, the target background is assumed to be triggered by a coincidence of dark hits and small clusters of hits due to radioactive sources in the FRP or the structure of the detector wall.

We can separate this background from the solar neutrino signal by searching for a small cluster in both space and time. A real neutrino signal at the edge region also has similar characteristics, but it causes a bigger cluster compared to the background events. Thus, the key is to evaluate the size of the hit cluster.

In order to check the size of the hit cluster for an event, a new cut variable was created. This variable is a product of two factors. One factor is the radius of a circle which contains the clustered hits in an event, and the other is the number of clustered hits within a $20 \mathrm{nsec}$ time window. Figure 17 shows the distribution of this cut variable for real events and for solar neutrino MC simulation events in a energy region 5.0 to $6.5 \mathrm{MeV}$. For this figure, a volume cut with $r>12 \mathrm{~m}$ and $z>-3 \mathrm{~m}$ is applied to study background events in the barrel, which are due to FRP cover radioactivity.

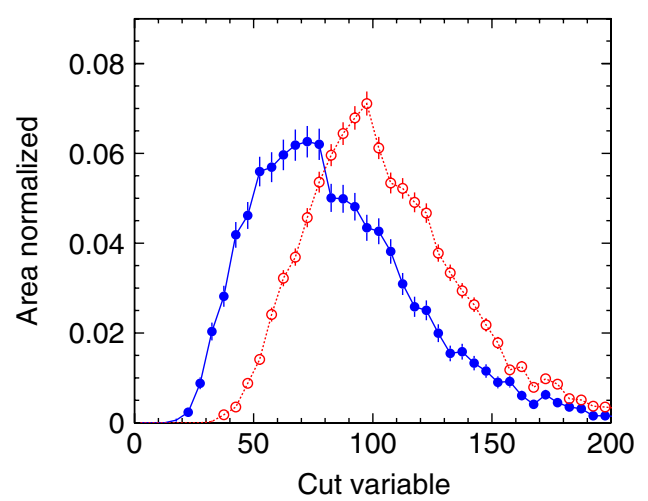

FIG. 17 (color online). Distribution of cut variable for the small clustered hit cut. Blue (filled circle) shows background sample events which are selected from $r>12 \mathrm{~m}$ and $z>-3 \mathrm{~m}$. Red (open circle) shows the solar neutrino MC simulation and the same volume cut is applied. For both data and MC events, event selection cuts up to the external event cut are applied. The reconstructed energies of these samples are in 5.0-6.5 MeV. 
As expected, the real data sample which contains mostly FRP events shows smaller values for this cut variable than simulated solar neutrino events. A cut value of 75 is selected which gives maximum significance (Signal $/ \sqrt{\mathrm{BG}}$ ) for the solar neutrino signal. This cut is applied to events with energy $<6.5 \mathrm{MeV}$ whose vertex position is in an edge region. The edge region is determined for $5.0-5.5 \mathrm{MeV}$, and 5.5-6.5 MeV energy bins separately, to optimize the significance of the solar neutrino signal. The criteria of the edge region are determined as follows:

$$
\begin{aligned}
\left\{r^{2}>180 \mathrm{~m}^{2}\right. & \text { for } 5.5 \leq E<6.5 \mathrm{MeV} \\
r^{2}>155 \mathrm{~m}^{2} & \text { for } 5.0 \leq E<5.5 \mathrm{MeV}
\end{aligned}
$$

where $r$ is defined in Fig. 3. Figure 18 shows the vertex distribution of the final data sample for the 5.0-6.5 MeV energy region before and after the small clustered hits cut.

\section{Tighter fiducial volume cut}

This cut is to reject the remaining background in the edge region. As shown in Fig. 19, background events in the bottom region still remain in the final data sample. This nonuniformity of the vertex distribution of the background distorts the angular distribution of the background, which causes a large systematic uncertainty for the day-night asymmetry of solar neutrino flux.

To set a tight fiducial volume, the significance as a function of detector radius is calculated. Based on the significance calculation, the final value of fiducial volume is obtained for each energy region.

$$
\begin{gathered}
5.0-5.5 \mathrm{MeV}:\left(r^{2}<180 \mathrm{~m}^{2}\right. \text { and } \\
z>-7.5 \mathrm{~m})=13.3 \mathrm{kton}
\end{gathered}
$$

\section{5-20 MeV: no tight fiducial volume cut $=22.5 \mathrm{kton}$}

Note that in the future, we hope to remove this tighter fiducial volume cut by improving our signal extraction

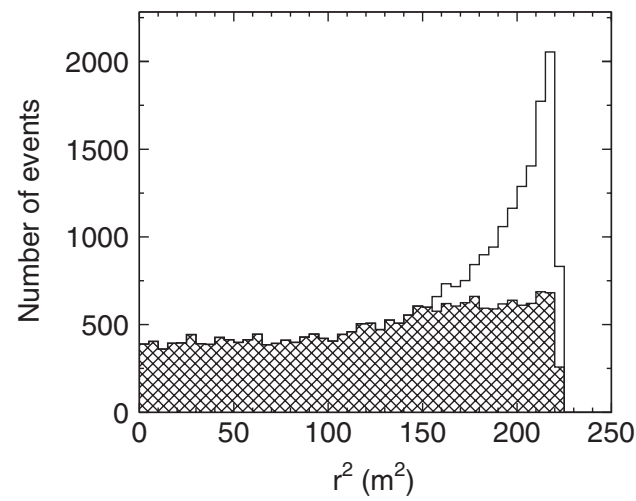

FIG. 18. Vertex distribution of 5.0-6.5 MeV energy region before and after the small cluster hits cut. The horizontal axis shows $r^{2}$ and the vertical axis shows the number of events. Events with $z>-7 \mathrm{~m}$ are selected to show background events in the barrel.

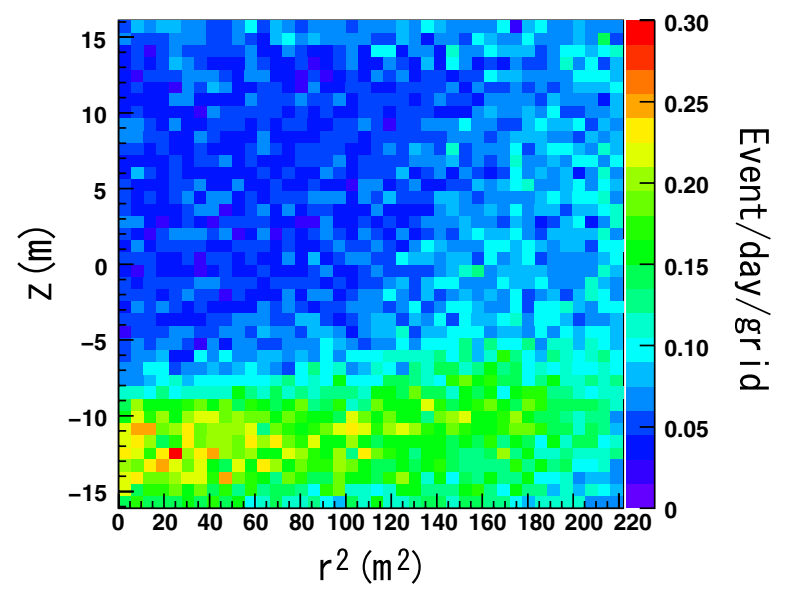

FIG. 19 (color online). Vertex distribution of final data sample in the 5.0-6.5 MeV energy region (22.5 kton fiducial volume).

method, because solar neutrino events are preferentially rejected in the low energy region by this cut.

\section{E. Summary of event selection}

Figure 20 shows the energy spectrum after each step of the reduction and Fig. 21 shows the remaining efficiency of ${ }^{8} \mathrm{~B}$ solar neutrino MC with respect to the reconstructed energy. The number of events after each reduction step is summarized in Table II (real data) and Table III (solar neutrino MC simulation). While the event rate in the real data as a function of energy is the same as for SK-I, the cut efficiencies are improved by $10 \%$ in the final SK-III data sample.

\section{F. Simulation of solar neutrinos}

The method to extract the energy spectrum and the flux of solar neutrinos in SK-III is based on that of SK-I and II.

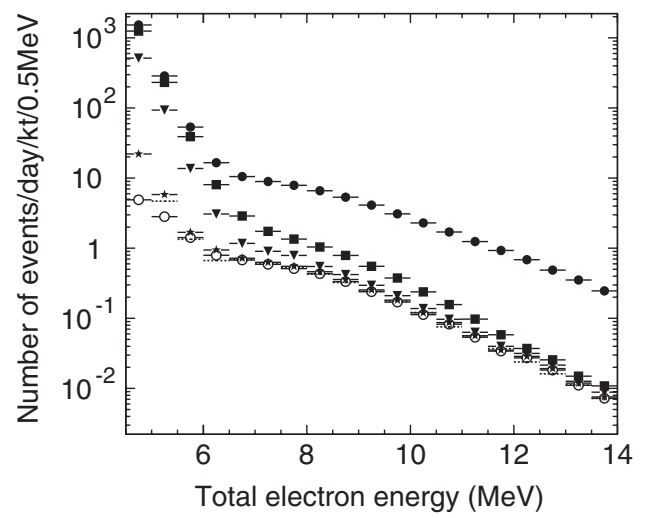

FIG. 20. Number of events after each event reduction step as a function of energy. From the top, the markers show events after spallation cut, event quality cut, external event cut, ${ }^{16} \mathrm{~N}$ cut, and small clustered hit cut plus tight fiducial volume cut. The dashed histogram shows the number of events in the SK-I final sample. 


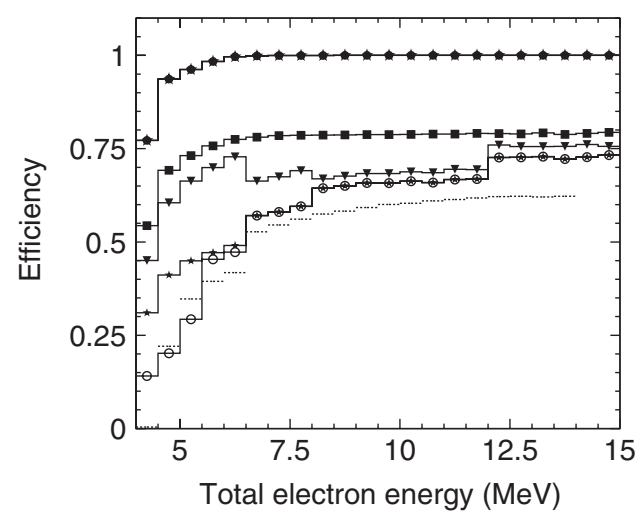

FIG. 21. Reduction efficiency for the solar neutrino MC simulation. The definition of each histogram is the same as for Fig. 20.

The ${ }^{8} \mathrm{~B}$ and hep neutrino spectra are generated separately. The total ${ }^{8} \mathrm{~B}$ and hep flux values are referred from the SSM [5]. We use the spectrum and its uncertainty of ${ }^{8} \mathrm{~B}$ and hep neutrinos calculated by Winter [12] and Bahcall [13], respectively. The event time of a solar neutrino event is simulated from the live time of the SK-III full operation period, so that the expected zenith angle distribution of the solar neutrinos can be simulated correctly.

TABLE II. Reduction results for data.

\begin{tabular}{lcc}
\hline \hline Reduction step & $5.0-6.5 \mathrm{MeV}$ & $6.5-20.0 \mathrm{MeV}$ \\
\hline Spallation cut & 1861770 & 114350 \\
Event quality cuts & 734843 & 58187 \\
External event cut & 54820 & 43146 \\
${ }^{16} \mathrm{~N}$ cut & 54351 & 39879 \\
Small cluster hits cut & 42916 & \\
Tight fidv. cut & 24311 & 39879 \\
Final & 24311 & \\
\hline \hline
\end{tabular}

TABLE III. Reduction results for solar neutrino MC events in $\%$. For the spallation cut and ${ }^{16} \mathrm{~N}$ cut, position-dependent dead time is considered.

\begin{tabular}{lcc}
\hline \hline Reduction step & $5.0-6.5 \mathrm{MeV}$ & $6.5-20.0 \mathrm{MeV}$ \\
\hline Total & 100 & 100 \\
Bad run cut & 86 & 87 \\
Trigger condition & 72 & 86 \\
Flasher events cut & 56 & 67 \\
Spallation $+{ }^{16} \mathrm{~N}$ cut & 45 & 53 \\
Event quality cuts & 45 & 46 \\
External event cut & 28 & 42 \\
Small cluster hits cut & 27 & \\
Tight fidv cuts & 24 & 42 \\
Final & 24 & \\
\hline \hline
\end{tabular}

\section{G. Systematic uncertainty}

The following items are updated with respect to SK-I with the improved calibration, detector simulation and analysis tools described above.

(i) Angular resolution

In order to estimate the systematic uncertainty on the total flux due to the angular resolution difference between data and MC simulation, a predicted energy spectrum is made by artificially shifting the reconstructed direction of the solar neutrino MC events. The shifted direction is calculated as the reconstructed direction \pm the systematic uncertainty of angular resolution with respect to the generated direction of the recoil electron. After the solar angle fitting, the systematic uncertainty due to the uncertainty of angular resolution is estimated as $\pm 0.67 \%$ on the total flux in $5.0-20 \mathrm{MeV}$ region $( \pm 1.2 \%$ for SK-I). This method of estimation is the same as for SK-I, so the improvement is due to the new detector simulation and angular reconstruction.

(ii) Vertex shift and resolution

The vertex shift difference between data and MC is used to estimate the systematic uncertainty of the fiducial volume. We measure this difference using $\mathrm{Ni}$ calibration rather than the LINAC, because LINAC electrons always point downwards while solar neutrino recoil electrons go in all directions. Also, very few positions near the edge of the fiducial volume can be probed by the LINAC. As an example, Fig. 22 shows the observed vertex shift difference as a function of energy at $(15.2 \mathrm{~m}$, $-0.7 \mathrm{~m}, 12 \mathrm{~m}$ ), where the vertex shift in the $x$ direction is larger than other positions in Fig. 2. There is no evidence that the vertex shift difference is energy dependent.

To estimate the total flux uncertainty due to the vertex shift, the reconstructed vertex positions of

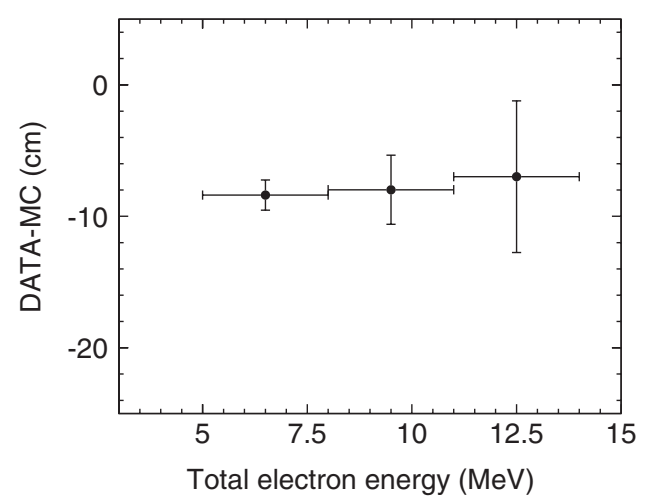

FIG. 22. The vertex shift as a function of energy. The markers show the amount of vertex shift measured by Ni calibration at $x=15.2 \mathrm{~m} y=-0.7 \mathrm{~m}$ and $z=12 \mathrm{~m}$. For the vertical axis, negative values mean vertex shifts inward towards the center of the detector. 
the solar neutrino MC events are artificially shifted outward. Then, the fraction of events rejected by the fiducial volume cut due to the shift is estimated. Figure 23 shows the systematic uncertainty on the fiducial volume due to the vertex shift which is based on the results of the Ni calibration. Note that in SK-I and SK-II, the estimation was done by a calculation based on the size of the vertex shift, whereas in SK-III, MC simulations after event selection are used to take into account the cuts' efficiencies at the edge of the fiducial volume. This new method gives a more accurate estimation.

This study shows that $0.54 \%$ of the MC events are rejected after shifting the vertex position, and this fraction is set to the total flux systematic uncertainty due to the vertex shift ( $\pm 1.3 \%$ for SK-I). The improvement with respect to SK-I is mainly due to the reduction of the vertex shift.

In Fig. 23, the step in the fiducial volume uncertainty between 5.0 and $6.0 \mathrm{MeV}$ is due to the tight fiducial volume cut for the lower energy region $(<5.5 \mathrm{MeV})$. This relative difference of fiducial volume systematic uncertainty is taken into account in the uncertainty of the energy spectrum shape. Between 5.5 and $20 \mathrm{MeV}$, since vertices are assumed to be shifted in the same direction for all energies, the relative differences are $0.1 \%$. Consequently, the systematic uncertainty of the spectrum shape is set to $0.1 \%$ in that energy region. In the $5.0-5.5 \mathrm{MeV}$ region, the systematic error is set to $0.5 \%$ because the uncertainty of the fiducial volume is relatively $0.5 \%$ larger than for the other region.

The vertex resolution is also compared for data and MC simulation using LINAC events. Figure 24 shows the difference of vertex resolution as a function of energy. This difference results in only a second order effect on the systematic uncertainty of the fiducial volume.

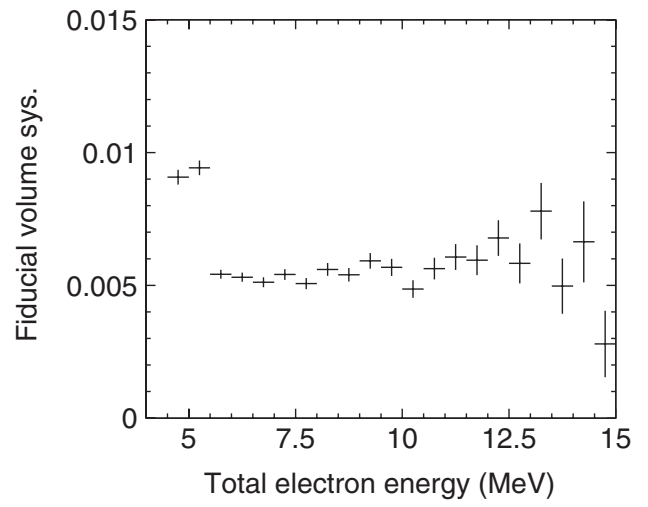

FIG. 23. The systematic uncertainty of the fiducial volume as a function of energy.

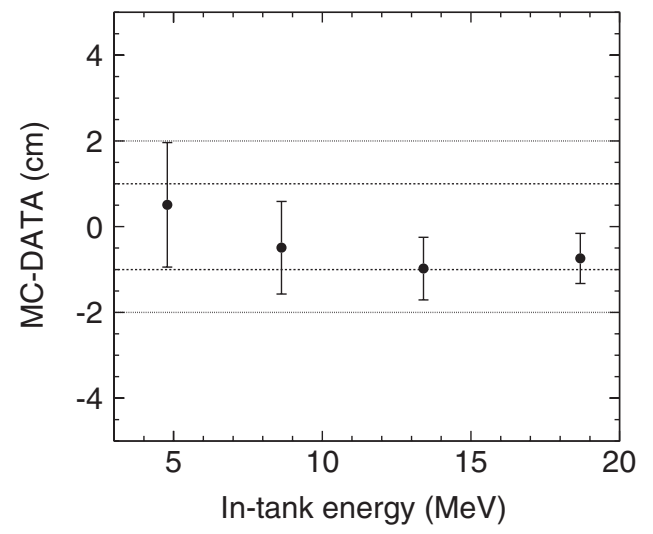

FIG. 24. The difference of vertex resolution between MC simulation and data as a function of energy obtained by LINAC calibration. The dashed line and the dotted line show $1 \mathrm{~cm}$ and $2 \mathrm{~cm}$ difference, respectively.

(iii) Event quality cuts

The systematic uncertainties associated with the event quality cuts are estimated separately for (a), (b) and (c) of Sec. III B. For (a) and (b), the systematic uncertainties on the total flux are taken from the differences of the efficiencies between the LINAC data and MC simulation which are $\pm 0.4 \%$ and $\pm 0.25 \%$, respectively.

For (c), the second vertex cut, the estimation is done in the same way as for the first vertex fitter, resulting in a $0.45 \%$ contribution to the total flux uncertainty.

The combined uncertainty is $0.65 \%$ which is about one third of SK-I's. The improvement is due to the improved event selection methods and better-tuned MC simulation.

(iv) Small clustered hits cut Using DT data and MC simulation at the position $(x=-12 \mathrm{~m}, y=0 \mathrm{~m}, z=0 \mathrm{~m})$, a difference in cut efficiency between DT data and MC events of

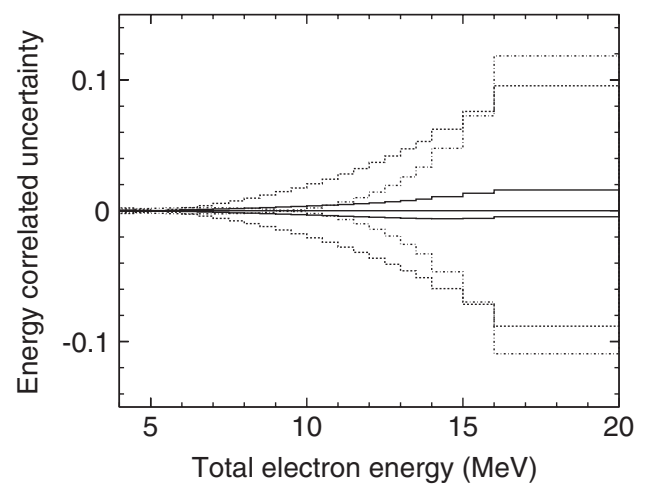

FIG. 25. Energy-correlated systematic uncertainties. The solid, dotted, and dashed lines show the uncertainties of the ${ }^{8} \mathrm{~B}$ spectrum, the energy scale, and the energy resolution, respectively. 
$2 \%$ is obtained. Considering the entire fiducial volume and the energy region of the solar neutrino signal, this difference corresponds to a $0.5 \%$ uncertainty on the total flux in the $5.0-20 \mathrm{MeV}$ region. A value of $2 \%$ is assigned to the spectral shape uncertainty for the 5.0 to $6.5 \mathrm{MeV}$ bins.

(v) Signal extraction method

The solar neutrino flux is obtained by fitting a solar angle distribution (see Fig. 26). To check biases of the fitting method to the flux value, the solar angle fit is applied to dummy data which have known numbers of signal and background events. As a result, $\pm 0.7 \%$ difference is found between the input and the output number of signal events for the total flux, and $\pm 2 \%$ difference is found especially in the $5.0<$ $E<5.5 \mathrm{MeV}$ region.

\section{Summary of systematic uncertainties}

(i) Flux, time variation, day-night asymmetry

The systematic uncertainties on total flux, time variation and day-night asymmetry are summarized in Table IV. The systematic uncertainty on the total flux is estimated to be $2.1 \%$. This is about two thirds of the corresponding SK-I value. The main contributions to the improvement are the vertex shift, angular resolution, and event selection uncertainties, which are reestimated for SK-III.

(ii) Spectrum

The systematic uncertainty of the spectral shape consists of two components:

(a) Energy-correlated:

The energy-correlated systematic uncertainties are obtained by counting the number of events in the solar neutrino MC simulation with artificially shifted energy scale, energy resolution and ${ }^{8} \mathrm{~B} \nu$ energy spectrum. The results of the calculations

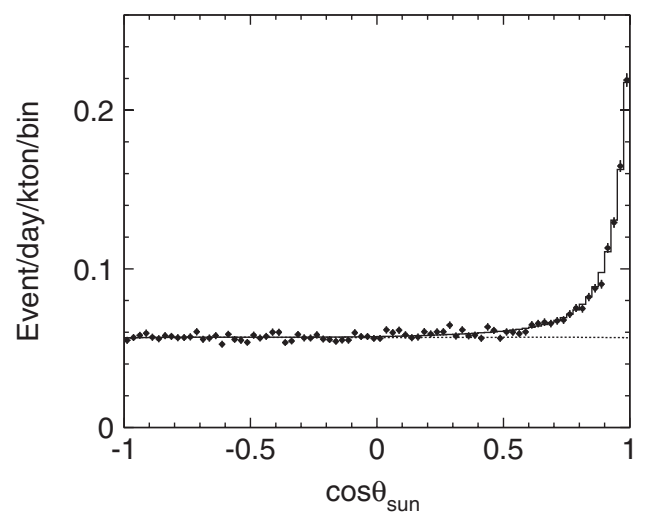

FIG. 26. The angular distribution of the solar neutrino final sample events. The dotted line seen under the peak in the solar direction represents background contributions.
TABLE IV. Summary of the systematic uncertainty of the total flux in $\%$.

\begin{tabular}{lc}
\hline \hline Source & Total Flux \\
\hline Energy scale & \pm 1.4 \\
Energy resolution & \pm 0.2 \\
${ }^{8}$ B spectrum & \pm 0.2 \\
Trigger efficiency & \pm 0.5 \\
Angular resolution & \pm 0.67 \\
Fiducial volume (vertex shift) & \pm 0.54 \\
Event quality cuts & \\
- Quality cut & \pm 0.4 \\
- Hit pattern cut & \pm 0.25 \\
- Second vertex & \pm 0.45 \\
Spallation & \pm 0.2 \\
External event cut & \pm 0.25 \\
Small cluster hits cut & \pm 0.5 \\
Background shape & \pm 0.1 \\
Signal extraction method & \pm 0.7 \\
Live time & \pm 0.1 \\
Cross section & \pm 0.5 \\
Total & \pm 2.1 \\
\hline \hline
\end{tabular}

are shown in Fig. 25. These correlations are taken into account in the oscillation analysis.

(b) Energy-uncorrelated:

The energy-uncorrelated spectral uncertainties are listed in Table V. To obtain the effect on the energy spectral shape, relative differences of each uncertainty between each energy bin are studied. For example, the uncertainty of the fiducial volume due to the vertex shift is $0.54 \%$ in total, and the relative differences are obtained as $0.1 \%$ for 5.5-20 MeV region, but $0.5 \%$ for $5.0-5.5 \mathrm{MeV}$ region with the tight fiducial volume. These uncertainties are taken into account in the oscillation analysis without correlations.

\section{H. Total flux result}

Recoil electrons from elastic solar $\nu$-electron scattering are strongly forward-biased. SK-III statistically separates solar $\nu$ 's from background with an unbinned likelihood fit to the directional distribution with respect to the Sun. For a live time of 548 days of SK-III data, from 5.0 to $20.0 \mathrm{MeV}$, the extracted number of signal events is $8132+133-$ 131 (stat) \pm 186 (sys). The corresponding ${ }^{8} \mathrm{~B}$ flux is obtained using the ${ }^{8} \mathrm{~B}$ spectrum of [12] to be:

$$
(2.32 \pm 0.04 \text { (stat) } \pm 0.05 \text { (sys) }) \times 10^{6} \mathrm{~cm}^{-2} \mathrm{sec}^{-1} \text {. }
$$

This result is consistent with SK-I $(2.38 \pm 0.02$ (stat) \pm 0.08 (sys) $\left.\times 10^{6} \mathrm{~cm}^{-2} \mathrm{sec}^{-1}\right) \quad$ and SK-II $\quad(2.41 \pm$ 0.05 (stat) ${ }_{-0.15}^{+0.16}(\mathrm{sys}) \times 10^{6} \mathrm{~cm}^{-2} \mathrm{sec}^{-1}$ ). The SK-I and II values are recalculated using the ${ }^{8} \mathrm{~B}$ spectrum of [12].

Figure 26 shows the angular distribution of extracted solar neutrino events. 
TABLE V. Energy-uncorrelated systematic uncertainty on the observed spectrum shape in $\%$.

\begin{tabular}{lllllll}
\hline \hline Energy (MeV) & $5-5.5$ & $5.5-6$ & $6-6.5$ & $6.5-7$ & $7-7.5$ & $7.5-20$ \\
\hline Trig eff & \pm 2.4 & \pm 0.9 & \pm 0.1 & $\ldots$ & $\ldots$ & $\ldots$ \\
(a) & \pm 2. & \pm 1.75 & \pm 1.5 & \pm 1.25 & \pm 1.0 & $\ldots$ \\
(b) & $\ldots$ & $\ldots$ & $\ldots$ & \pm 0.25 & \pm 0.25 & \pm 0.25 \\
Small cluster hits cut & \pm 2. & \pm 2. & \pm 2. & $\ldots$ & $\ldots$ & $\ldots$ \\
External event cut & \pm 0.1 & \pm 0.1 & \pm 0.1 & \pm 0.1 & \pm 0.1 & \pm 0.1 \\
Fiducial volume (vertex shift) & \pm 0.5 & \pm 0.1 & \pm 0.1 & \pm 0.1 & \pm 0.1 & \pm 0.1 \\
BG shape & \pm 0.2 & \pm 0.8 & \pm 0.2 & \pm 0.2 & \pm 0.2 & \pm 0.2 \\
Sig. Ext. & \pm 2.1 & \pm 0.7 & \pm 0.7 & \pm 0.7 & \pm 0.7 & \pm 0.7 \\
Cross section & \pm 0.2 & \pm 0.2 & \pm 0.2 & \pm 0.2 & \pm 0.2 & \pm 0.2 \\
Total & \pm 4.3 & \pm 3.0 & \pm 2.6 & \pm 1.5 & \pm 1.3 & \pm 0.8 \\
\hline \hline
\end{tabular}

TABLE VI. SK-III observed energy spectra expressed in units of event/kton/year. The errors in the observed rates are statistical only. The expected rates neglecting oscillation are for the BP2004 SSM flux values. $\theta_{z}$ is the angle between the $z$-axis of the detector and the vector from the Sun to the detector.

\begin{tabular}{|c|c|c|c|c|c|}
\hline \multirow[t]{2}{*}{ Energy $(\mathrm{MeV})$} & \multirow[b]{2}{*}{$\mathrm{ALL}-1 \leq \cos \theta_{\mathrm{z}} \leq 1$} & \multirow{2}{*}{$\begin{array}{c}\text { Observed rate } \\
\text { DAY }-1 \leq \cos \theta_{\mathrm{z}} \leq 0\end{array}$} & \multicolumn{3}{|c|}{ Expected rate } \\
\hline & & & NIGHT $0<\cos \theta_{z} \leq 1$ & ${ }^{8} \mathrm{~B}$ & hep \\
\hline $5.0-5.5$ & $83.3_{-10.0}^{+10.3}$ & $94.6_{-15.0}^{+15.8}$ & $73.5_{-13.1}^{+13.7}$ & 193.4 & 0.334 \\
\hline $5.5-6.0$ & $67.9_{-6.2}^{+6.4}$ & $75.2_{-9.4}^{+9.8}$ & $61.5_{-8.0}^{+8.5}$ & 177.0 & 0.321 \\
\hline $6.0-6.5$ & $63.5_{-4.8}^{+5.0}$ & $55.9_{-6.6}^{+7.0}$ & $71.0_{-6.7}^{+7.1}$ & 160.4 & 0.310 \\
\hline $6.5-7.0$ & $55.3_{-2.6}^{+2.7}$ & $51.3_{-3.7}^{+3.9}$ & $59.1_{-3.7}^{+3.9}$ & 139.7 & 0.289 \\
\hline $7.0-7.5$ & $54.0_{-2.4}^{+2.5}$ & $55.9_{-3.5}^{+3.7}$ & $52.3_{-3.4}^{+3.5}$ & 121.9 & 0.271 \\
\hline $7.5-8.0$ & $40.6_{-2.1}^{+2.2}$ & $39.9_{-3.0}^{+3.2}$ & $41.2_{-2.9}^{+3.1}$ & 105.8 & 0.257 \\
\hline $8.0-8.5$ & $36.7_{-1.8}^{+1.9}$ & $37.5_{-2.6}^{+2.8}$ & $35.9_{-2.5}^{+2.6}$ & 89.8 & 0.240 \\
\hline $8.5-9.0$ & $30.9_{-1.6}^{+1.7}$ & $28.7_{-2.2}^{+2.4}$ & $32.9_{-2.3}^{+2.4}$ & 75.0 & 0.223 \\
\hline $9.0-9.5$ & $22.6_{-1.3}^{+1.4}$ & $20.0_{-1.8}^{+1.9}$ & $25.2_{-1.9}^{+2.1}$ & 61.8 & 0.205 \\
\hline $9.5-10.0$ & $19.5_{-1.2}^{+1.3}$ & $18.0_{-1.6}^{+1.8}$ & $20.8_{-1.7}^{+1.8}$ & 49.5 & 0.186 \\
\hline $10.0-10.5$ & $14.5_{-1.0}^{+1.0}$ & $15.2_{-1.4}^{+1.5}$ & $13.8_{-1.3}^{+1.5}$ & 39.2 & 0.169 \\
\hline $10.5-11.0$ & $14.0_{-0.9}^{+1.0}$ & $15.2_{-1.3}^{+1.5}$ & $13.0_{-1.2}^{+1.3}$ & 30.3 & 0.151 \\
\hline $11.0-11.5$ & $9.62_{-0.74}^{+0.81}$ & $9.67_{-1.07}^{+1.21}$ & $9.56_{-1.00}^{+1.12}$ & 22.76 & 0.134 \\
\hline $11.5-12.0$ & $5.74_{-0.58}^{+0.65}$ & $5.33_{-0.78}^{+0.92}$ & $6.17_{-0.83}^{+0.96}$ & 16.81 & 0.118 \\
\hline $12.0-12.5$ & $5.01_{-0.52}^{+0.58}$ & $4.20_{-0.68}^{+0.81}$ & $5.77_{-0.74}^{+0.86}$ & 12.09 & 0.102 \\
\hline $12.5-13.0$ & $3.11_{-0.39}^{+0.45}$ & $2.74_{-0.50}^{+0.63}$ & $3.47_{-0.55}^{+0.67}$ & 8.44 & 0.088 \\
\hline $13.0-13.5$ & $1.97_{-0.30}^{+0.36}$ & $1.63_{-0.36}^{+0.49}$ & $2.30_{-0.44}^{+0.56}$ & 5.56 & 0.074 \\
\hline $13.5-14.0$ & $1.37_{-0.24}^{+0.30}$ & $1.17_{-0.28}^{+0.40}$ & $1.53_{-0.36}^{+0.48}$ & 3.70 & 0.062 \\
\hline $14.0-15.0$ & $2.22_{-0.31}^{+0.37}$ & $2.08_{-0.41}^{+0.53}$ & $2.35_{-0.43}^{+0.54}$ & 3.74 & 0.092 \\
\hline $15.0-16.0$ & $0.866_{-0.182}^{+0.243}$ & $0.394_{-0.164}^{+0.298}$ & $1.266_{-0.288}^{+0.404}$ & 1.285 & 0.059 \\
\hline $16.0-20.0$ & $0.117_{-0.067}^{+0.136}$ & $0.252_{-0.121}^{+0.245}$ & $0.000_{-0.422}^{+0.130}$ & 0.570 & 0.068 \\
\hline
\end{tabular}

\section{Energy spectrum}

The recoil electron energy spectrum is obtained from 5.0 to $20.0 \mathrm{MeV}$ in 21 bins. The definition of the energy bins is given in Table VI, which shows the observed and expected event rates. Figure 27 shows the observed energy spectrum divided by the SSM (BP2004) [5] without oscillation. The line in the
Fig. 27 represents the total SK-III average (flat data/ SSM prediction without neutrino oscillation effect). The $\chi^{2}$ value is $27.1 / 20$ dof for the flat prediction and 26.8/20 dof for the prediction with the best-fit neutrino oscillation parameters obtained by the global solar analysis (see Sec. IVC). This result indicates no significant spectral distortion. 


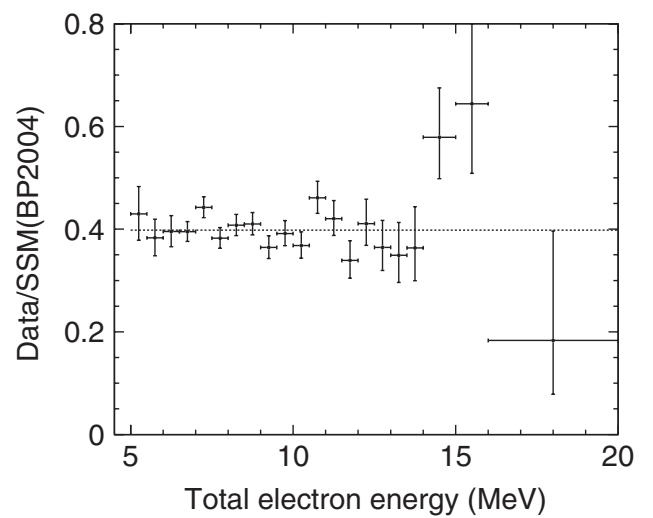

FIG. 27. Ratio of observed and expected energy spectra. The dashed line represents the SK-III average.

\section{OSCILLATION ANALYSIS WITH SK-III RESULT}

Oscillations of solar neutrinos have been studied by numerous experiments. The results from such experiments have placed increasingly stringent constraints on the mixing angle between neutrino mass and flavor eigenstates as well as on the neutrino mass difference.

In Sec. IV B, the result of the two-flavor neutrino oscillation analysis using SK-I, SK-II, SK-III data is presented. A conventional two-flavor analysis is done in order to compare directly with previous results. The results from all other solar neutrino experiments and KamLAND are combined in Sec. IV C. The two-flavor analysis in SK-III is accomplished in basically the same way as the previous SK-I and SK-II analyses. Updates to the experimental data which are used in this analysis are explained in each section.

In Sec. IV D, the results of the three-flavor analysis are shown. The experimental data have been improved statistically and systematically and now have a few percent uncertainty. In this paper, we present the first result of a three-flavor analysis using full SK data information.

\section{A. $\chi^{2}$ definition}

The oscillation analysis of SK uses the spectrum, time variation (zenith angle dependence), and total flux in determination of the solar neutrino oscillation parameters $\left(\theta_{12}, \Delta m_{12}\right)$. For each set of oscillation parameters, the total ${ }^{8} \mathrm{~B}$ and hep neutrino fluxes are fit to the data. The entire SK-III observed spectrum is utilized for a $5.0 \mathrm{MeV}$ threshold. The MSW [14] $\nu_{e}$ survival probabilities are numerically calculated from the solar matter distribution provided by $\operatorname{SSM}(\mathrm{BP} 2004)$. The absolute ${ }^{8} \mathrm{~B}$ and hep neutrino flux predictions of the SSM are only used as a normalization. The uncertainty of the ${ }^{8} \mathrm{~B}$ neutrino flux of the SSM is not used in the calculation of the $\chi^{2}$. We use the uncertainty of the SNO (NC) measurement instead (see the next section); hence, the absolute ${ }^{8} \mathrm{~B}$ flux predicted by the SSM does not affect the fitted ${ }^{8} \mathrm{~B}$ neutrino flux value. For the hep neutrino flux, we use the uncertainty of the SSM, since experimental uncertainty is still large; hence the fitted hep neutrino flux is constrained by the SSM prediction. The predicted neutrino spectrum is then converted to an expected SK-III rate spectrum by using the $\nu-e$ elastic scattering cross section and the SK-III detector energy resolution. To account for the systematic uncertainties in energy resolution as well as the energy scale and the ${ }^{8} \mathrm{~B}$ neutrino spectrum model shape, the combined rate predictions are modified by energy shape factors, $f\left(E_{i}, \delta_{B}, \delta_{S}, \delta_{R}\right)$. The quantities $\delta_{B}, \delta_{S}$, and $\delta_{R}$ represent uncertainty in the ${ }^{8} \mathrm{~B}$ neutrino spectrum, SK-III energy scale, and SK-III energy resolution, respectively. The function $f$ serves to shift the rate predictions corresponding to a given uncertainty $\delta$ in the data rate. The following equation describes the SK-III spectrum $\chi^{2}$ along with energycorrelated systematic error shape factors applied to the expected rate:

$$
\begin{aligned}
\chi_{\mathrm{SK}-\mathrm{III}}^{2}= & \sum_{i=1}^{21} \frac{\left(d_{i}-\left(\beta b_{i}+\eta h_{i}\right) \times f\left(E_{i}, \delta_{B}, \delta_{S}, \delta_{R}\right)\right)^{2}}{\sigma_{i}^{2}} \\
& +\left(\frac{\delta_{B}}{\sigma_{B}}\right)^{2}+\left(\frac{\delta_{S}}{\sigma_{S}}\right)^{2}+\left(\frac{\delta_{R}}{\sigma_{R}}\right)^{2}+2 \Delta \log (L) \\
& +\frac{\left(\phi_{\mathrm{SNO}}-\beta\right)^{2}}{\sigma_{\mathrm{SNO}}^{2}}+\frac{(1-\eta)^{2}}{\sigma_{\mathrm{hep}}^{2}},
\end{aligned}
$$

where $d_{i}$ is the observed rate divided by the expected, unoscillated rate for the $i$ th energy bin. Similarly, $b_{i}$ and $h_{i}$ are the predicted MSW oscillated rates divided by the unoscillated rate for ${ }^{8} \mathrm{~B}$ and hep neutrinos, respectively. $\beta$ $(\eta)$ scales the ${ }^{8} \mathrm{~B}$ (hep) neutrino flux. $\mathcal{L}$ is the unbinned time-variation likelihood for the SK-III solar zenith angle flux variation above a 5.0 MeV threshold. This likelihood is analogous to the one used in SK-I and SK-II. The last two terms in the Eq. (4.1) are the constraining terms of ${ }^{8} \mathrm{~B}$ and hep fluxes, respectively. The value of $\phi_{\mathrm{SNO}}$ is the $\mathrm{NC}$ value coming from the measurement of SNO divided by the SSM flux value, and $\sigma_{\text {SNO }}$ is the accompanying uncertainty of their measurements $[3,4]$. The numerical values are $\phi_{\mathrm{SNO}}=0.899$ and $\sigma_{\mathrm{SNO}}=0.032 . \sigma_{\text {hep }}$ is the uncertainty of SSM prediction on the hep neutrino flux, which is $16 \%$ [5].

\section{B. Oscillation results-SK with constrained flux}

The oscillation analysis is performed by including $\chi^{2}$ terms corresponding to the SK-I and SK-II values (namely, the spectrum and unbinned time variation for SK-I and SKII). By constraining the ${ }^{8} \mathrm{~B}$ flux to the total $\mathrm{NC}$ flux value from SNO, allowed parameter regions can be obtained. Figure 28 shows allowed regions at $95 \%$ confidence level. The result is consistent with previous SK-I and SK-II results. This result is the first to show that the energy spectrum and the time variation of the solar neutrino flux 


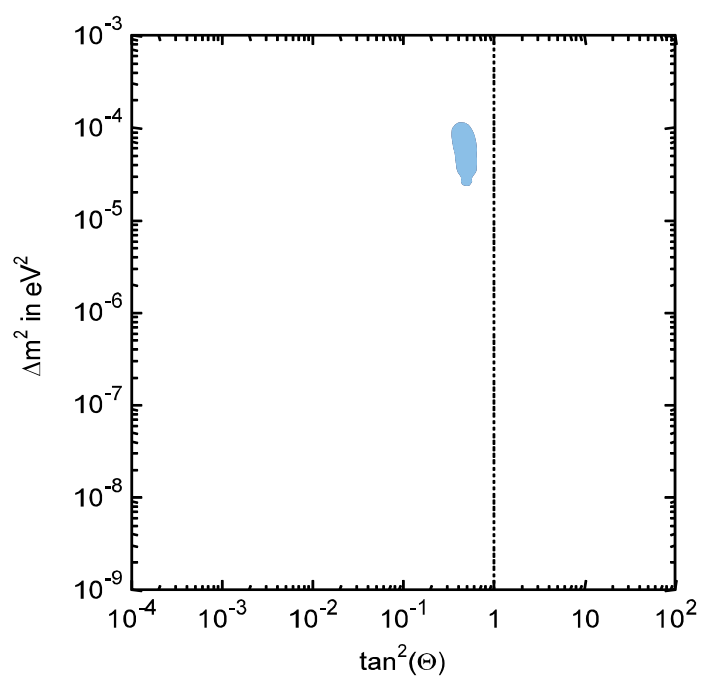

FIG. 28 (color online). $95 \%$ C.L. allowed region from SK-I, II, III combined analysis. The ${ }^{8} \mathrm{~B}$ flux is constrained by the SNO NC rate (LETA and phase-III).

measured by SK favor only LMA solution at $95 \%$ C.L., by constraining the ${ }^{8} \mathrm{~B}$ neutrino flux to the SNO NC flux and the hep neutrino flux to the SSM prediction.

\section{Combined oscillation results from several experiments}

The combination of other solar neutrino experimental results such as the SNO, Borexino and radiochemical results with the SK combined analysis is accomplished with a two-flavor neutrino oscillation analysis by constructing a global $\chi^{2}$. For the SNO results, the total charged current (CC) rates observed in the 306-day pure $\mathrm{D}_{2} \mathrm{O}$ phase (SNO-I) [15], 391-day salt phases (SNO-II) [16], and 385day NCD phase (SNO-III) [3], the combined NC rates of LETA [4] and SNO-III, and the predicted day-night asymmetry for SNO-I and II are used. In this analysis, the correlations between the SNO CC and NC rates are not taken into account. The ${ }^{7} \mathrm{Be}$ solar neutrino flux of Borexino's 192-day [17] and the radiochemical experiments of Homestake [18], GALLEX-GNO [19], and stratospheric aerosol and gas experiment [20] are then included into the global $\chi^{2}$ with the fluxes and their correlations calculated by SSM in a way shown by [21]. Figure 29 shows the combined solar allowed region. The best-fit parameter set is $\sin ^{2} \theta_{12}=0.30_{-0.01}^{+0.02}\left(\tan ^{2} \theta_{12}=0.42_{-0.02}^{+0.04}\right)$ and $\Delta m_{21}^{2}=6.2_{-1.9}^{+1.1} \times 10^{-5} \mathrm{eV}^{2}$, consistent with the SK-I global analysis. In addition, combining the above and KamLAND data, the best-fit parameter set is $\sin ^{2} \theta_{12}=$ $0.31 \pm 0.01\left(\tan ^{2} \theta_{12}=0.44 \pm 0.03\right) \quad$ and $\quad \Delta m_{21}^{2}=$ $7.6 \pm 0.2 \times 10^{-5} \mathrm{eV}^{2}$ as shown in Fig. 30 .

Figure 31 shows the $95 \%$ allowed region for all solar experiments before and after the SK-III result is included. As shown in the figure, the SK-III result contributes about $5 \%$ improvement to the uncertainty of $\Delta m_{12}^{2}$.

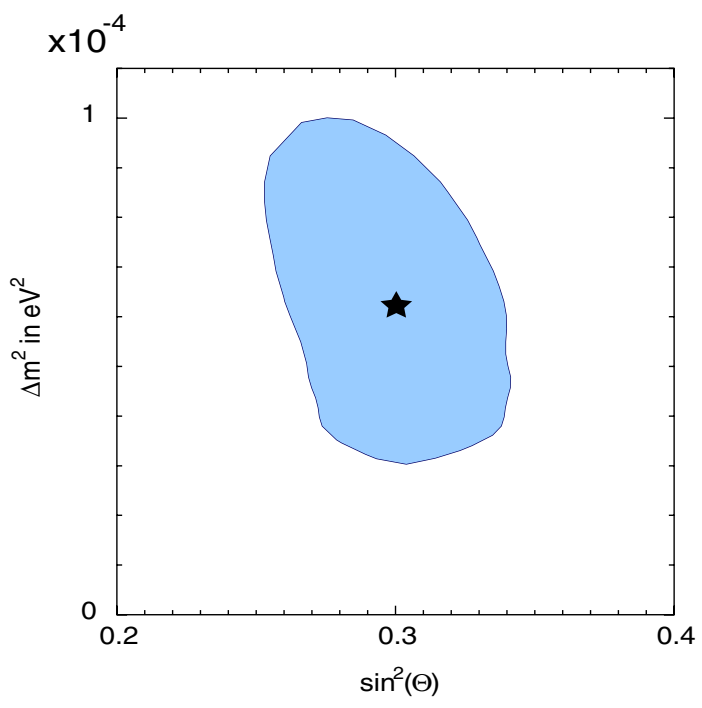

FIG. 29 (color online). Allowed region for all solar experiments at $95 \%$ C.L..

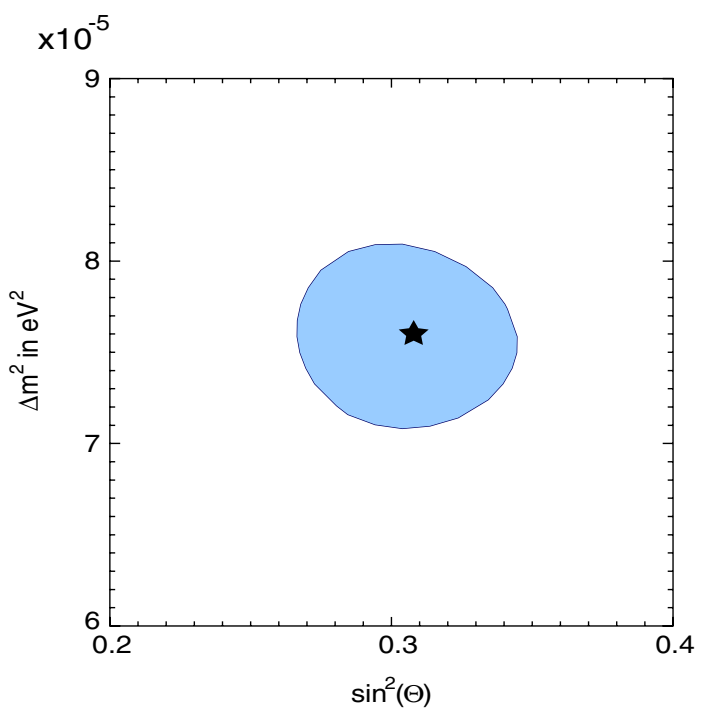

FIG. 30 (color online). Allowed region for all solar experiments and KamLAND for two-flavor analysis at 95\% C.L..

\section{Three-flavor analysis}

In a three-flavor analysis, the calculation of oscillation probability is based on [22]. The probability can be calculated with three parameters: $\theta_{12}, \theta_{13}$, and $\Delta m_{12}^{2}$, assuming $\Delta m_{12}^{2} \ll \Delta m_{23}^{2} \sim \Delta m_{13}^{2}$. We fixed $\Delta m_{23}^{2}=$ $2.4 \times 10^{-3} \mathrm{eV}^{2}$ and the normal hierarchy is assumed. For the solar neutrino oscillation, the other mixing parameters are irrelevant, but we set $\theta_{23}=\pi / 4$ and $\delta_{C P}=0$ in our calculation.

As done for the two-flavor analysis, the oscillation probabilities depending on different zenith angles are calculated, and then the rate of the radiochemical experiments, Borexino ( ${ }^{7} \mathrm{Be}$ neutrino flux), and SNO (CC 


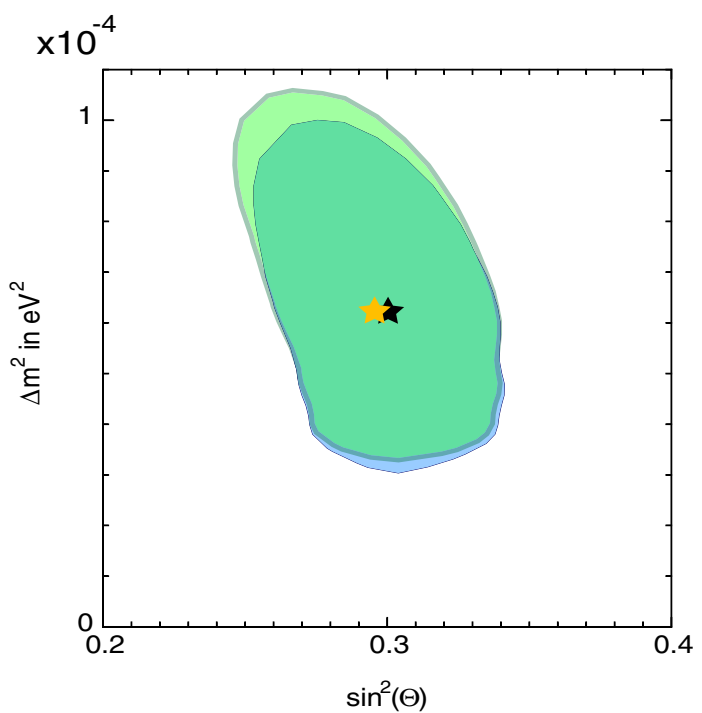

FIG. 31 (color online). Allowed region for all solar experiments for two-flavor analysis at 95\% C.L.. The light green contour and the yellow star show the result without SK-III, and the light blue contour and the black star show the result with SK-III, which is same as Fig. 29.

for all phases, NC for LETA and phase-III) are calculated. SNO day-night asymmetries for phase I and II are also predicted.

The KamLAND spectra and rates from surrounding reactors are calculated based on the information from the KamLAND official database [23] and the published paper [24]. Because the information in [23] is used for their second result [25], we normalized the calculated neutrino spectrum without oscillation to that of Figure 1 of [24]. The oscillation probability is calculated using three-flavor vacuum oscillation. The amount of background and systematic uncertainties for each energy bin are read from Figure 1 of [24]. The background is fixed in our calculation of $\chi^{2}$. The systematic uncertainty for each energy bin is treated as an energy-uncorrelated systematic uncertainty. The systematic uncertainty of event rate on Table 1 of [24] is also taken into account. We checked first that the contour of the two-flavor analysis in Figure 2 of [24] was reproduced with $\theta_{13}=0$. We also checked that our three-flavor contour was very close to the contour presented in [26], and consistent with their latest result [27].

The oscillation parameters are scanned in the following regions: $\quad 10^{-5} \mathrm{eV}^{2}<\Delta m_{12}^{2}<2 \times 10^{-4} \mathrm{eV}^{2}, \quad 0.1<$ $\tan ^{2} \theta_{12}<1.0$, and $0<\sin ^{2} \theta_{13}<0.25$. Figure 32 shows the allowed region of the solar neutrino parameters, $\left(\theta_{12}, \Delta m_{12}^{2}\right)$, obtained by the three-flavor analysis of the global solar results. The allowed region for KamLAND obtained by our three-flavor analysis and the allowed region for global solar and KamLAND combined analysis are also shown in Fig. 32. Inclusion of another oscillation parameter $\theta_{13}$ results in a weaker constraint on the solar parameter space.

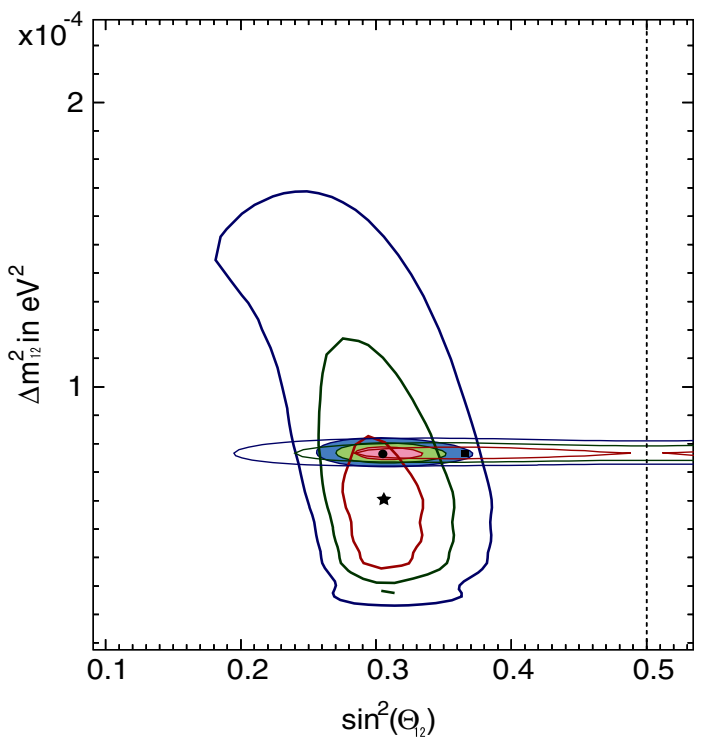

FIG. 32 (color online). Allowed region in solar parameter space $\left(\theta_{12}, \Delta m^{2}\right)$ obtained by the three-flavor analysis. The thick lines and the star mark show the allowed regions and the best-fit point of the global solar analysis. The thin lines and the square mark show the allowed regions and the best-fit point of our KamLAND analysis. The filled areas and the filled circle mark show the allowed regions and the best-fit point of the combined analysis. For all regions, the innermost area (red), the middle area (green) and the outermost area (blue) show 68.3, 95, 99.7\% C.L., respectively.

Figure 33 shows the allowed region in $\left(\theta_{12}, \theta_{13}\right)$ space obtained from the global solar analysis and our KamLAND analysis. As shown in the figure, in the global solar contour, the larger value of $\theta_{13}$ prefers the larger value of $\theta_{12}$, while in the KamLAND contour

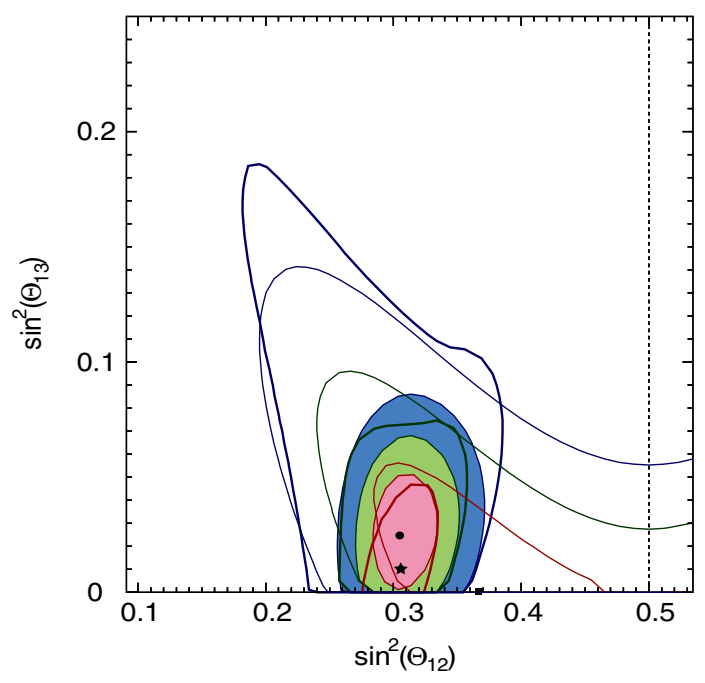

FIG. 33 (color online). Allowed region in $\left(\theta_{12}, \theta_{13}\right)$ space obtained by the three-flavor analysis. The definitions of marks and lines are same as in Fig.c 32. 
TABLE VII. ${ }^{8} \mathrm{~B}$ neutrino flux obtained from the oscillation parameter fitting.

\begin{tabular}{lc}
\hline \hline & ${ }^{8} \mathrm{~B}$ flux $\left(\times 10^{6} \mathrm{~cm}^{-2} \mathrm{~s}^{-1}\right)$ \\
\hline Global solar (2 flavor) & $5.3 \pm 0.2$ \\
Global solar + KamLAND (2 flavor) & $5.1 \pm 0.1$ \\
Global solar (3 flavor) & $5.3 \pm 0.2$ \\
Global solar + KamLAND (3 flavor) & $5.3_{-0.2}^{+0.1}$ \\
\hline \hline
\end{tabular}

the larger value of $\theta_{13}$ prefers the smaller value of $\theta_{12}$. The global solar analysis finds that the best-fit values at $\sin ^{2} \theta_{12}=0.31 \pm 0.03\left(\tan ^{2} \theta_{12}=0.44 \pm 0.06\right)$ and $\Delta m_{21}^{2}=$ $6.0_{-2.5}^{+2.2} \times 10^{-5} \mathrm{eV}^{2}$. Combined with the KamLAND result, the best-fit oscillation parameters are found to be $\sin ^{2} \theta_{12}=0.31_{-0.02}^{+0.03}\left(\tan ^{2} \theta_{12}=0.44_{-0.04}^{+0.06}\right)$ and $\Delta m_{21}^{2}=$ $7.7 \pm 0.3 \times 10^{-5} \mathrm{eV}^{2}$. The best-fit value of $\sin ^{2} \theta_{13}$ is 0.01 , and an upper bound is obtained, $\sin ^{2} \theta_{13}<0.060$ at the $95 \%$ C.L., for the global solar analysis. Combining with the KamLAND contour, the best-fit value of $\sin ^{2} \theta_{13}$ is $0.025_{-0.016}^{+0.018}$ and the $95 \%$ C.L. upper limit of the $\sin ^{2} \theta_{13}$ is found to be 0.059 .

The flux value of ${ }^{8} \mathrm{~B}$ neutrinos can be extracted using the oscillation parameters obtained from the fitting of the global solar and KamLAND result. As in Eq. (4.1), $\beta$ is a free parameter to minimize the $\chi^{2}$ and there is no constraint from the SSM prediction in $\chi_{\mathrm{SK}+\mathrm{SNO}}^{2}$. Table VII summarizes the scaled ${ }^{8} \mathrm{~B}$ flux values by using $\beta_{m}$ at the best-fit point obtained by the global solar analysis and the global solar + KamLAND analyses in both two and threeflavor analyses. The size of the error corresponds to the maximum and minimum flux values among the $1 \sigma$ oscillation parameter region. As shown in the table, the ${ }^{8} \mathrm{~B}$ flux agrees well with the latest SSM prediction [28], and the size of the uncertainty is $2 \sim 3 \%$ which is consistent with the $\mathrm{SNO}$ result [4].

\section{CONCLUSION}

Super-Kamiokande has measured the solar ${ }^{8} \mathrm{~B}$ flux to be $(2.32 \pm 0.04$ (stat) \pm 0.05 (sys) $) \times 10^{6} \mathrm{~cm}^{-2} \mathrm{sec}^{-1}$ during its third phase; the systematic uncertainty is smaller than for SK-I. Combining all solar experiments in a twoflavor fit, the best fit is found to favor the LMA region at $\sin ^{2} \theta_{12}=0.30_{-0.01}^{+0.02}\left(\tan ^{2} \theta_{12}=0.42_{-0.02}^{+0.04}\right)$ and $\Delta m_{21}^{2}=$ $6.2_{-1.9}^{+1.1} \times 10^{-5} \mathrm{eV}^{2}$. Combined with the KamLAND result, the best-fit oscillation parameters are found to be $\sin ^{2} \theta_{12}=0.31 \pm 0.01\left(\tan ^{2} \theta_{12}=0.44 \pm 0.03\right)$ and $\Delta m_{21}^{2}=$ $7.6 \pm 0.2 \times 10^{-5} \mathrm{eV}^{2}$, in excellent agreement with previous solar neutrino oscillation measurements. In a threeflavor analysis combining all solar neutrino experiments and the KamLAND result, the best-fit value of $\sin ^{2} \theta_{13}$ is found to be $0.025_{-0.016}^{+0.018}$ and an upper bound is obtained as $\sin ^{2} \theta_{13}<0.059$ at $95 \%$ C.L..

\section{ACKNOWLEDGMENTS}

The authors gratefully acknowledge the cooperation of the Kamioka Mining and Smelting Company. Super-K has been built and operated from funds provided by the Japanese Ministry of Education, Culture, Sports, Science and Technology, the U.S. Department of Energy, and the U.S. National Science Foundation. This work was partially supported by the Research Foundation of Korea (BK21 and KNRC), the Korean Ministry of Science and Technology, the National Science Foundation of China, and the Spanish Ministry of Science and Innovation (Grants No. FPA200913697-C04-02 and No. Consolider-Ingenio-2010/CPAN).
[1] J. Hosaka et al., Phys. Rev. D 73, 112001 (2006).

[2] J.P. Cravens et al., Phys. Rev. D 78, 032002 (2008).

[3] B. Aharmim et al., Phys. Rev. Lett. 101, 111301 (2008).

[4] B. Aharmim et al., Phys. Rev. C 81, 055504 (2010).

[5] J. N. Bahcall and M. H. Pinsonneault, Phys. Rev. Lett. 92, 121301 (2004).

[6] M. Smy, in Proceedings of the 30th International Cosmic Ray Conference, edited by Rogelio Caballero et al. (Universidad Nacional Autónoma de México, Mexico City, 2008, Vol. 5, p. 1279-1282.

[7] S. Fukuda et al., Nucl. Instrum. Methods Phys. Res., Sect. A 501, 418 (2003).

[8] M. Nakahata et al., Nucl. Instrum. Methods Phys. Res., Sect. A 421, 113 (1999).

[9] E. Blaufuss et al., Nucl. Instrum. Methods Phys. Res., Sect. A 458, 636 (2001).
[10] R. M. Pope and E. S. Fry, Appl. Opt. 36, 8710 (1997).

[11] R. M. Pops et al., Appl. Opt. 35, 33 (1997).

[12] W. T. Winter, S.J. Freedman, K.E. Rehm, and J.P. Schiffer, Phys. Rev. C 73, 025503 (2006).

[13] J. N. Bahcall et al., Nucl. Phys. B77, 64 (1999).

[14] S. P. Mikheyev and A. Y. Smirnov, Sov. J. Nucl. Phys. 42, 913 (1985); L. Wolfenstein, Phys. Rev. D 17, 2369 (1978).

[15] B. Aharmim et al., Phys. Rev. C 75, 045502 (2007).

[16] S. N. Ahmed et al., Phys. Rev. C 72, 055502 (2005).

[17] C. Arpesella et al., Phys. Rev. Lett. 101, 091302 (2008).

[18] B. T. Cleveland et al., Astrophys. J. 496, 505 (1998).

[19] M. Altmann et al., Phys. Lett. B 490, 16 (2000); also presented at Neutrino 2004 by C. Cattadori.

[20] J. N. Abdurashitov et al., Phys. Rev. C 60, 055801 (1999).

[21] G. L. Fogli, E. Lisi,A. Marrone, D. Montanino, and A. Palazzo, Phys. Rev. D 66, 053010 (2002). 
[22] V. Barger, K. Whisnant, S. Pakvasa, and R. J. N. Phillips, Phys. Rev. D 22, 2718 (1980).

[23] KamLAND official data base wep page: http://www.awa .tohoku.ac.jp/KamLAND/datarelease/2ndresult.html

[24] S. Abe et al., Phys. Rev. Lett. 100, 221803 (2008).

[25] T. Araki et al., Phys. Rev. Lett. 94, 081801 (2005).
[26] K. Ichimura KamLAND collaboration, 34th International Conference on High Energy Physics (ICHEP 2008) arXiv:0810.3448v1.

[27] A. Gando et al. The KamLand Collaboration, Phys. Rev. D 83, 052002 (2011).

[28] A. M. Serenelli et al., Astrophys. J. Lett. (to be published). 